Themenheft Nr. 40: CoViD-19 und die digitale Hochschulbildung. Irritationen, Einsichten und Programmatiken

Herausgegeben von Markus Deimann, Marios Karapanos und Klaus Rummler

\title{
Digitales Lehren und Lernen im Corona- Semester aus der Sicht von Bachelor- und Masterstudierenden
}

\author{
Konsequenzen für eine agile Qualitätsentwicklung der Hochschullehre \\ Kristina Kögler ${ }^{1}$ (D), Florina Ștefănică ${ }^{1}$, Christine Sälzer ${ }^{1}$ D , Stefan Behrendt ${ }^{1}$ (D), \\ Marlene Scherfer ${ }^{1}$ (D) und Suemeyye Atlihan ${ }^{1}$ \\ ${ }^{1}$ Universität Stuttgart
}

\begin{abstract}
Zusammenfassung
Die pandemiebedingte, kurzfristige Umstellung der Hochschullehre auf ausschliesslich digitale Veranstaltungsangebote stellte Lehrende und Studierende im Frühjahr 2020 vor grosse Herausforderungen. Unter enormem Zeitdruck mussten bewährte Präsenzlehrformate transformiert und für Lehrende wie Studierende organisationale Unterstützungssysteme etabliert werden. Dieser forcierte Digitalisierungsprozess beinhaltet für hiesige Hochschulen die Chance, die Weiterentwicklung der Lehre agil und evidenzbasiert voranzutreiben und dabei nicht zuletzt auch die Herausforderungen und Grenzen digitalen Lernens zu identifizieren. Nachhaltige Digitalisierungsstrategien sollten ganzheitlich und mehrperspektivisch sein, hier bietet insbesondere der Einbezug der Perspektive von Studierenden wichtige Einsichten für den Digitalisierungsprozess und die Qualitätsentwicklung der Hochschullehre. Diesem Beitrag liegen die Fragen zugrunde, wie Studierende die Rahmenbedingungen, Qualität und Effekte der digitalen Hochschullehre im ersten Corona-Semester 2020 bewerten und mit welchen spezifischen Herausforderungen sie sich konfrontiert sehen. Erkenntnisse dazu wurden an der Universität Stuttgart im Rahmen einer Fragebogenerhebung in drei Wellen gewonnen. Es zeigt sich etwa, dass die Strukturierung der Arbeit zuhause vielen Studierenden schwerfällt und die gefühlte Arbeitsbelastung gestiegen ist. Besonders Bachelorstudierende wünschen sich mehr Unterstützung bei der Vorbereitung auf Prüfungen und der Koordination von Lerngruppen. Neben der Ableitung von konkreten Optimierungsempfehlungen stehen insbesondere die Konsequenzen im Fokus, die sich für die strategische Qualitätsentwicklung der Hochschullehre und den weiteren Digitalisierungsprozess ergeben.
\end{abstract}


Digital Teaching and Learning in the Corona Semester from the Perspective of Students. Consequences for an Agile Quality Development

\begin{abstract}
The pandemic-related, short-term conversion of university teaching to the digital confronted lecturers and students with major challenges in spring 2020. Under enormous time pressure, proven teaching formats had to be transformed and organizational support systems had to be established for both teachers and students. This accelerated digitization process offers universities the opportunity to drive forward the further development of teaching in an agile and evidence-based manner and to identify the challenges and limitations of digital learning. Sustainable digitization strategies should be holistic and multi-perspective, especially including the perspective of students offers important insights for the digitization process and the quality development of university teaching. This paper is based on the questions of how students evaluate the conditions, quality and effects of the digital teaching in the first Corona Semester 2020 and what specific challenges they face. Findings were obtained at the University of Stuttgart as part of a questionnaire survey in three waves. Many students find it difficult to structure their work at home and their perceived workload has increased. Especially bachelor students need more support in preparing for exams and coordinating learning groups. Consequences for the strategic quality development of university teaching and the digitization process are taken in view.
\end{abstract}

\title{
1. Ausgangslage
}

Im Frühjahr 2020 musste der hochschulische Lehrbetrieb aufgrund der Covid-19-Pandemie in kürzester Zeit auf rein digitale Lehre umgestellt werden. Die Transformation etablierter Präsenzlehrformate unter grösstem Zeitdruck und der ausschliesslich virtuelle Austausch über Studieninhalte stellte alle hochschulischen Akteure, nicht zuletzt Lehrende und Studierende, vor grosse Herausforderungen und führte in vielen Hochschulen zu umfänglichen organisatorischen, infrastrukturellen sowie hochschuldidaktischen Unterstützungsmassnahmen sowie Reflexionsprozessen, die vielfach auch durch empirische Evidenzen in Gestalt verschiedener Studien flankiert wurden (z. B. Hafer, Kostädt, und Lucke 2020; Skulmowski und Rey 2020; Rapanta et al. 2020). Angesichts dessen, dass die Frage der Digitalisierung der Hochschullehre auch schon vor Pandemiebeginn rege diskutiert worden war (vgl. Adedoyin und Soykan 2020; Kopp, Gröblinger, und Adams 2019) und in der Regel über einen konzeptionellen Konsens bezüglich des Wünschbaren und prinzipiell Möglichen bisher nicht nennenswert hinausging (Wannemacher 2017), beinhaltet der jüngst forcierte Transformationsprozess für hiesige Hochschulen nicht zuletzt die Gelegenheit, die 
Weiterentwicklung der Lehre agil und evidenzbasiert voranzutreiben (Lehner und Volk 2018) und dabei gezielt auch die Herausforderungen und Grenzen digitalen Lernens zu identifizieren (Leimeister und David 2019; Zierer 2018), wobei hier naturgemäss die konzeptionell wichtige Unterscheidung zwischen dem aktuellen «Emergency Remote Teaching» und regulären onlinebasierten Lehr-Lern-Formaten gemässigt zu berücksichtigen ist (s. etwa Hodges et al. 2020). Erste aktuelle Befunde in diesem Kontext weisen zumeist anhand von querschnittlichen Zugängen bei insgesamt mässig positiver Bilanz auf diverse Problemlagen auf Seiten der Studierenden hin - es zeichnet sich spezifischer Unterstützungsbedarf ab (z. B. Mulders und Krah 2021), der aus Forschungsperspektive nahelegt, insbesondere sozio-emotionale, infrastrukturelle und organisatorische Ausgangsbedingungen und Entwicklungen zu untersuchen (Brahm und Pumptow 2021).

Der Auftrag von Hochschulen besteht nicht nur darin, eine qualitativ hochwertige akademische Ausbildung anzubieten, sondern auch die bestmögliche Kompetenzentwicklung für alle Studierenden in den unterschiedlichen Studiengängen und -profilen zu unterstützen. Dies ist eine sehr anspruchsvolle Aufgabe, die sowohl didaktische, methodische als auch politische und gesellschaftliche Gegebenheiten und Anforderungen sowie die Bedürfnisse und Eingangsvoraussetzungen unterschiedlicher Kohorten in Bachelor- und Masterstudiengängen einbeziehen muss (Euler und Seufert 2005). Gleichzeitig sind die Strukturen an deutschen Hochschulen bisweilen zu starr und Veränderungen werden nur verhältnismässig langsam umgesetzt. Dementsprechend ist die Hochschulbildung in Deutschland, im Gegensatz zu Ländern im angloamerikanischen Sprachraum oder in Skandinavien, noch nicht umfangreich digital transformiert (Handke 2020). Zwar nutzen die Lehrenden häufig digitale Tools, um Inhalte zu teilen oder Informationen zu verwalten, aber grosse Teile der Lehre fanden bis dato immer noch im bewährten Präsenzmodus und unter Nutzung einer geringen Bandbreite an Anwendungen und Technologien statt (Schmid et al. 2017). Es stellt sich naturgemäss insbesondere in Präsenzhochschulen die Frage nach der sinnvollen Intensität und Ausgestaltung einer pädagogisch und curricular zielführenden Flankierung und Anreicherung der Lehre um digitale Funktionalitäten.

In diesem Kontext zeichnet sich ein Bedarf an Forschung zu den Sichtweisen von Lehrenden wie auch Studierenden ab, der in jüngerer Zeit stärker adressiert wurde (z. B. Dittler und Kreidl 2020). In dem umfangreichen Diskurs zur digitalen Transformation in der Hochschullandschaft besteht weitgehender Konsens darüber, dass nachhaltige und zielführende Digitalisierungsstrategien ganzheitlich angelegt und mehrperspektivisch sein sollten (Seufert et al. 2015). Die Beschränkung der handelnden Akteure auf Hochschulleitung, Lehrende, IT-Support und Qualitätsentwicklung erscheint nicht zielführend. Vielmehr bietet auch und insbesondere der Einbezug der Perspektive von Studierenden wichtige Einsichten für den Digitalisierungsprozess und die Qualitätsentwicklung der Hochschullehre, nicht zuletzt in 
systemakkreditierten Hochschulen (Suwalski 2020). Der Stellenwert des Einbezugs der Perspektiven von Bachelor- und Masterstudierenden ist im Zuge der disruptiven Ad-Hoc-Transformation noch einmal deutlich gestiegen.

Der Fokus des vorliegenden Beitrags liegt daher auf den Ergebnissen einer Studierendenbefragung, die während des Sommersemesters 2020 in drei Wellen durchgeführt wurde und Teil des umfassenden Evaluationsprojekts "CorUS» zur Erfassung der «Effekte der Corona-Pandemie auf Lehrqualität und Studienerfolg an der Universität Stuttgart» ist, welche zu den grössten technischen Universitäten in Deutschland (TU9) zählt und von daher einen gewissen Modellcharakter für andere Standorte aufweisen dürfte. Die Studierenden wurden dabei zu ihren Erwartungen und Urteilen bezüglich der Ausgestaltung des digitalen Lehrens und Lernens befragt. Berücksichtigt wurden auch Fragen der technischen Ausstattung und des häuslichen Lernumfelds sowie gefühlte Effekte der veränderten Lehr-Lern-Situation auf Wohlbefinden, Studienzufriedenheit und (selbsteingeschätzten) Studienerfolg. Nach einigen grundsätzlichen Überlegungen zur Digitalisierung der Hochschullehre als strategischer Herausforderung und dem Stellenwert von studentischen Rückmeldungen im Rahmen der Qualitätsentwicklung der Hochschullehre werden ausgewählte empirische Ergebnisse aus den drei Erhebungswellen vorgestellt und hinsichtlich ihrer hochschuldidaktischen und strategischen Implikationen diskutiert.

\section{Ad-Hoc-Digitalisierung der Hochschullehre als strategische Herausforderung}

Die Potenziale, Herausforderungen und notwendigen Rahmenbedingungen der Digitalisierung in Bildungseinrichtungen werden mit den voranschreitenden technologischen Möglichkeiten seit mehreren Jahrzehnten breit diskutiert und beforscht. Insbesondere auch in Universitäten und Hochschulen für angewandte Wissenschaften sind sie Gegenstand umfangreicher strategischer, pädagogisch-didaktischer und organisatorischer Erwägungen. Der Begriff der Digitalisierung der Bildung wird durch Kerres (2016) als «eine Kurzformel für den grundliegenden Transformationsprozess der Bildungsarbeit, der - anders als E-Learning - die gesamte Wertschöpfung der Wissenserschliessung und -kommunikation in den Blick nimmt verstanden. Entscheidendes Charakteristikum ist, dass es nicht mit einem schlichten «Ergänzen` von Lernangeboten um soziale und mobile Komponenten des Lernens getan ist, sondern, dass neue Geschäftsmodelle entstehen und veränderte Leistungsprozesse nötig sind (Dittler 2017) - es muss sich insofern eine echte digitale Transformation vollziehen, die alle Stakeholder in die Lage versetzt, "die Chancen der Digitalisierung und von Netzwerkeffekten für die Hochschulentwicklung selbständig und eigenverantwortlich nutzen zu können» (Seufert, Guggemos, und Moser 2019, 89). Es sind davon also nicht nur einzelne Akteursgruppen, sondern alle - Hochschulleitung, Hochschulverwaltung, IT-Support, Lehrende wie auch Studierende - gleichermassen betroffen 
und vor die Aufgabe gestellt, mit Blick auf die institutionellen Ziele und vor dem Hintergrund der jeweiligen Rahmenbedingungen Lösungen zu entwickeln und auszugestalten (z. B. Brahm et al. 2016; Getto und Kerres 2017). Ungeachtet der terminologischen Vielfalt und Diskussion darüber, was unter dem Schlagwort der Digitalisierung in welcher konzeptionellen Breite zu fassen sei (s. etwa Hofhues et al. 2020), stellt sich für Hochschulen insbesondere in medienpädagogischer und hochschuldidaktischer Hinsicht die Frage nach der bestmöglichen Ausgestaltung der Hochschullehre unter zielführender Rahmung durch organisationale Unterstützungssysteme. Dabei stehen schwerpunktmässig Fragen der pädagogischen oder didaktischen Qualität und der gezielten Förderung von Studierenden im Fokus - nicht zuletzt die sich aktuell vollziehende zweite Welle der Digitalisierung, die umfangreiche Möglichkeiten der Nutzung von adaptiven und intelligenten Lerntechnologien und -systemen mit sich bringt, induziert einen grossflächigen Bedarf an systematischen empirischen Erkenntnissen zu den Gelingensbedingungen und Effekten des (digitalen) Lehrens und Lernens in der Hochschule (Seufert, Guggemos, und Sonderegger 2020).

Der Status Quo der Digitalisierung an deutschen Hochschulen vor der disruptiven Ad-Hoc-Entwicklung im Zusammenhang mit der Covid-19-Pandemie im Frühjahr 2020 wird bislang allerdings eher zurückhaltend bis kritisch bilanziert (Schmid et al. 2017): Zwar gibt es in Deutschland umfangreiche bildungspolitische Initiativen - so hat die Bundesregierung etwa eine nationale digitale Agenda für die Jahre 2014 bis 2017 entwickelt, die alle Bildungsebenen adressiert, darüber hinaus existieren umfangreiche Forschungsförderprogramme des Bundes - die tatsächliche Konzeption und Umsetzung von ganzheitlichen Digitalisierungsstrategien befand sich allerdings zuletzt noch in einem eher frühen Stadium (vgl. Bond et al. 2018), es dominierten die klassischen Lehr-Lern-Formate, die allenfalls digital flankiert wurden (Schmid et al. 2017).

Allerdings haben Hochschulen im Vergleich etwa zu Schulen der Primar- und Sekundarstufe den entscheidenden Vorteil, dass sie nicht so zahlreich sind und in Bezug auf ihre digitale Infrastruktur ein höheres Mass an Autonomie geniessen. Lehrende und Studierende an Hochschulen konnten bereits zu Beginn der Covid-19-Pandemie auf eine im Bildungssektor vergleichsweise belastbare Ressourcenausstattung und entsprechende Routinen zurückgreifen. Gleichwohl waren Hochschulen wie Bildungssteuerung nicht auf die Wucht und Durchschlagskraft der kurzfristigen Ausschliesslichkeit digitaler Lehre vorbereitet. Der forcierte Transformationsprozess führte zwar zu einer sehr raschen und vollumfassend digitalen Ausgestaltung der Hochschullehre, es bleiben jedoch vielerlei Fragen offen. So ist etwa unklar, ob und inwiefern der Digitalisierungsprozess bestehende hochschulische Lehr-LernFormate in pädagogisch-didaktischer Hinsicht verändert hat. Zudem stellt sich in empirischer Hinsicht die Frage nach der Implementierungsqualität und Nutzung des Lehr-Lern-Angebots durch die Studierenden, die nur mithilfe empirischer Evidenzen zu adressieren ist. 
3. Studierende als Impulsgeber für die agile Weiterentwicklung der Lehrqualität Universitäten sind als Institutionen bereits mit ihrer Einrichtung legitimiert (Baecker 2017) und folgen innerhalb ihrer Rahmenbedingungen einer entsprechend institutionellen Logik. Spätestens mit der Umstellung der Curricula im Zuge der BolognaReform jedoch stösst das Selbstverständnis von Universitäten insofern an seine Grenzen, als auch sie sich den spezifischen Herausforderungen der kontinuierlichen Organisationsentwicklung stellen müssen. Transformationsprozesse können nicht durch blosses Aussitzen geschehen lassen werden, sie erfordern gezieltes Monitoring und strategische Steuerung, bestenfalls auf der Grundlage empirischer Evidenz. Im Gegensatz zur Institution sind Organisationen «problematische Formen der Ordnung von Arbeit, deren Sinn darin besteht, mit Alternativen verglichen zu werden (ebd., 20). Die Art und Weise, wie die Aufgaben einer Universität erfüllt werden, ist also mittlerweile begründungspflichtig und damit ein Managementthema. Universitäten schaffen ein Bildungsangebot und konkurrieren mit anderen Universitäten sowie anderen Hochschulen und Bildungseinrichtungen. Die Nutzer dieses Angebotes, also die Kundschaft, sind Studierende. Deren Ansprüche und Erwartungen, aber auch ihre Vorbildung sind heterogen und dynamisch. Gleichzeitig ist ihre Anwesenheit ein wichtiger Aspekt des universitären Budgets, der die Universität dazu motiviert, die eigenen Aufgaben so zu gestalten, dass neben den institutionellen Zielen auch die Ziele der Kundschaft erfüllt werden. Dies ist das Prinzip der Agilität im Management, das auch für die Organisationsentwicklung im universitären Bereich adäquat eingesetzt werden kann (Lehner und Volk 2018). Agil bedeutet in diesem Zusammenhang, pragmatisch und flexibel zu handeln - wozu die Universitäten aufgrund der pandemiebedingten Ad-hoc-Digitalisierung flächendeckend gezwungen waren.

Entscheidend für den Erfolg agiler Qualitätsentwicklungsprozesse ist die systematische und kurzfristige Sammlung empirischer Evidenz zur Unterstützung der strategischen Entscheidungen. Dementsprechend wurde das pandemiebedingt erste Digitalsemester an vielen Standorten begleitend evaluiert, indem bestehende Möglichkeiten zur Datenerhebung seitens der hochschulischen Qualitätsentwicklung genutzt und die gewonnenen Daten unter anderem mit Studierenden reflektiert und diskutiert wurden. Dies entspricht dem Prinzip, den Prozess aus Datenerhebung, Analyse und Reflexion sowie der Interpretation in Gesprächen mit Studierenden als Kundschaft sowie anderen zentralen Akteuren der Universität (Hochschulleitung, Studiendekane und Studiendekaninnen) rückzukoppeln (Lehner und Volk 2018). Studierende nehmen insofern die Rolle der reflektierenden Kundschaft ein und sind sowohl Datenquelle als auch Expertinnen und Experten bei der iterativen Interpretation der Auswertungen. Die entsprechenden Rückmelde- und Diskussionsprozesse wurden am Standort Stuttgart im Rahmen der regulären universitären Gremienarbeit und in einer Task Force digitale Lehre in engem Austausch mit den Studierendenvertretenden umgesetzt. 
Zur Einordnung der entsprechenden Rückläufe aus der Perspektive der Studierenden ist jedoch nicht zuletzt angesichts der hochschulpolitischen Tragweite der resultierenden Entscheidungen die Frage der Validität studentischer Urteile zu stellen. Aus der Forschung zur Evaluation der Hochschullehre ist bezüglich der Qualität studentischer Rückmeldungen mittlerweile gut belegt, dass die Urteile Studierender hinsichtlich ihrer Lehrveranstaltungen an der Universität Validitätsprobleme mit sich bringen können (etwa Benton und Cashin 2014; Rodabaugh und Kravitz 1994). So ist die Validität von Studierendenurteilen stets im Kontext der erfassten Variablen zu bewerten; insbesondere Merkmale des Kurses, der Lehrperson oder der Studierenden selbst und die Bekanntheit der Einschätzung anderer Kursteilnehmender zeigen regelmässig Effekte auf das vergebene Urteil. Das Feedback Studierender zu von innen besuchten hochschulischen Lehrveranstaltungen kann mit Barends, Rousseau und Briner $(2014,6)$ als «Stakeholder Evidence» klassifiziert werden und ist demnach immer auch von deren Erwartungen und Ansprüchen geprägt. Hier färbt vor allem die empfundene Fairness in einem Kurs die Wahrnehmung der Studierenden in Bezug auf absolvierte Lehrveranstaltungen ein. Die Einschätzung der Fairness hat sogar ein grösseres Gewicht als etwa die erzielte Note, der Arbeitsaufwand oder die Schwierigkeit des Kurses (Rodabaugh und Kravitz 1994). Zu bedenken ist jedoch neben Fragen der Validität auch eine meist eingeschränkte Reliabilität, die durch eine selektive Stichprobe vorliegt: Insbesondere im digitalen Setting dürfte die Datenbasis unvollständig sein, denn nur ein Teil der Kursteilnehmenden füllt den Evaluationsbögen aus - dies dürfte sich auch im aktuellen Setting nicht wesentlich verändern, bleibt aber eine empirische Frage.

Dennoch sind Studierende eine wichtige Quelle für Rückmeldungen und Einschätzungen hochschulischer Lehre, nicht zuletzt, da deren Einschätzungen Aufschluss für ihre Kompetenzentwicklung geben können und diese letztlich die Zielgrösse des Bildungsangebots markiert. Studierende sind die zentrale Zielgruppe der Universität und ihre Sicht auf eine der Kernaufgaben der Hochschulen ist ein notwendiger Input im Prozess der Qualitätsentwicklung. Gerade für systemakkreditierte Hochschulen ist eine entsprechende Evidenzbasierung nicht wegzudenken (Suwalski 2020): Studierenden kommt hier die Rolle als hochschulischen Akteuren zu, die im Qualitätsregelkreis kontinuierlich um ihr Urteil gebeten werden. Etwaige Validitätsprobleme lassen sich hier durch den multiperspektivischen Einbezug aller Statusgruppen, etwa auch der Lehrenden, zielführend adressieren. 


\section{Methodik und Stichprobe der Evaluationsstudie}

Um systematische Aussagen zu Rahmenbedingungen, Durchführungsqualität und den Effekten des digitalen Semesters treffen zu können, wurde an der Universität Stuttgart eine Fragebogenstudie durchgeführt, in der sowohl die Studierenden als auch die Lehrenden aller Fakultäten im Sommersemester 2020 in drei Erhebungswellen online schriftlich befragt wurden. Dabei wurden alle eingeschriebenen Studierenden sowie alle Lehrenden per E-Mail angeschrieben und um ihre Teilnahme an der Befragung gebeten, es handelt sich hier insofern um eine selbstselektive Stichprobenziehung. Im Rahmen des CorUS-Projekts werden auch hochschulstatistische Längsschnittdaten aggregiert ausgewertet und Beobachtungsdaten aus der digitalen Lernplattform ILIAS analysiert. Der vorliegende Beitrag fokussiert sich allerdings auf die Perspektive der Studierenden.

Zum ersten Erhebungszeitunkt (nach Beginn der Vorlesungszeit) wurden die Studierenden zu ihren Eingangsvoraussetzungen befragt. Dabei wurden unter anderem Soziodemographika, die IT-Ausstattung, das häusliche Arbeitsumfeld, Erwartungen an das Digitalsemester, Selbststeuerungsfähigkeit ${ }^{1}$ (Rheinberg und Wendland 2003), die IT-bezogene Selbstwirksamkeit (Egloffstein und Ifenthaler 2021; Schyns und von Collani 2002), das Studieninteresse ${ }^{2}$ (Krapp et al. 1993) oder die Vertrautheit mit verschiedenen Computeranwendungen (Richert et al. 2001) erhoben. Die zweite Erhebungswelle (kurz nach Ende der Vorlesungszeit) bezog sich auf Einschätzungen der Studierenden zu ihrem Lernprozess (Studierverhalten, Zufriedenheit mit digitaler Lehre, Zeitinvestments). Gegen Ende der Prüfungsphase wurde zu einem dritten Erhebungszeitpunkt nach der subjektiven Erfolgsbilanz und Erwartungen für das kommende Wintersemester gefragt; zusätzlich wurden das beständige Interesse und die Beharrlichkeit (GRIT-Skalen von Schmidt et al. 2019, Übersetzung von Duckworth et al. 2007) erfasst. Die Teilnahme erfolgte freiwillig und anonym, die Angaben werden über Codes gematcht, um Effekte über alle drei Erhebungswellen hinweg prüfen zu können.

An der ersten Befragung kurz nach Beginn der Vorlesungszeit nahmen $\mathrm{n}=2.602$ Studierende teil. Die Rücklaufquote betrug ca. $12 \%$ der etwa 22.200 angeschriebenen Studierenden über alle Fakultäten hinweg. Davon ordneten sich $47 \%$ dem weiblichen und $52 \%$ dem männlichen Geschlecht zu. Die Stichprobe ist geschlechtsbezogen nicht repräsentativ (Universität Stuttgart gesamt: $w=34 \%, M=66 \%$ ). Das Durchschnittsalter der Befragten liegt bei 23.03 Jahren ( $S D=4.32$ ). $54.4 \%$ der Teilnehmenden studieren in einem Bachelorstudiengang, $45.6 \%$ im Master. Bezogen auf die Verteilung in der Grundgesamtheit sind Bachelorstudierende in der Stichprobe leicht überrepräsentiert. Für $86 \%$ der Befragten war das Sommersemester 2020 die

1 Verwendete Skalen: Selbstkontrolle und Zielverfolgung, Selbstmotivierung und Emotionskontrolle, Prokrastination.

2 Verwendete Skalen: Gefühlsbezogene Valenzen, persönliche wertbezogene Valenzen, intrinsischer Charakter. 
erste Erfahrung mit rein digitaler Lehre, darunter sind etwas mehr Bachelor- als Masterstudierende. Über die zehn Fakultäten der Universität Stuttgart ${ }^{3}$ hinweg zeichnen sich nur geringfügige Unterschiede der Rücklaufquoten ab.

An der zweiten Erhebung kurz nach Ende der Vorlesungszeit nahmen $\mathrm{n}=2.132$ Studierende teil. Der Rücklauf war mit knapp $10 \%$ leicht rückläufig. $75 \%$ der Teilnehmenden hatten bereits an der ersten Befragung teilgenommen. Die Stichprobe enthält $40.4 \%$ weibliche Teilnehmende und $59.6 \%$ Teilnehmende männlichen Geschlechts - verglichen mit der Grundgesamtheit sind Frauen in der Stichprobe überrepräsentiert. Das Durchschnittsalter liegt nahezu unverändert bei $23.46 \mathrm{Jah}$ ren. $63.4 \%$ der Befragten studieren in einem Bachelorstudiengang. $36.6 \%$ sind in einem Master eingeschrieben. Die Mehrheit der Befragten lebte während des CoronaSemesters im Umkreis der Stadt Stuttgart, ein Drittel gab an, von weiter ausserhalb studiert zu haben.

Die dritte Befragung, die gegen Ende der Prüfungsphase durchgeführt wurde, beantworteten $n=2.059$ Studierende, die Rücklaufquote liegt damit bei $9.27 \% .47 .4 \%$ gaben an weiblichen Geschlechts, $52.3 \%$ männlichen Geschlechts zu sein. Das Durchschnittsalter lag bei 23.37 Jahren. Auch in der dritten Erhebungswelle waren Bachelorstudierende mit $\mathbf{6 2 . 2} \%$ der Teilnehmenden etwas überrepräsentiert. Tabelle 1 stellt die Verteilung der Studierenden über die drei Messzeitpunkte dar.

\begin{tabular}{|l|c|c|c|c|}
\hline & T1 & T2 & T3 & \\
\hline Nur T1 & 1188 & & & \\
\hline Nur T2 & & 717 & & \\
\hline Nur T3 & & & 722 & \\
\hline T1 und T2 & 397 & 397 & & \\
\hline T1 und T3 & 319 & & 319 & \\
\hline T2 und T3 & & 320 & 320 & \\
\hline T1, T2 und T3 & 698 & 698 & 698 & \\
\hline Gesamt pro Messzeitpunkt & 2602 & 2132 & 2059 & \\
\hline Gesamt im Datensatz & & & & 4361 \\
\hline
\end{tabular}

Tab. 1.: Verteilung der Studierenden über die drei Messzeitpunkte.

Nachfolgend werden ausgewählte explorative Ergebnisse aus allen drei Erhebungswellen berichtet: Dabei handelt es sich einerseits um querschnittliche Ergebnisse aus den einzelnen Wellen und andererseits um Ergebnisse, die auf Daten

3 Die Universität Stuttgart ist eine Universität mit technischem Fokus und gliedert sich in folgende Fakultäten: Fakultät 1: Architektur und Stadtplanung; Fakultät 2: Bau- und Umweltingenieurwissenschaften; Fakultät 3: Chemie; Fakultät 4: Energie-, Verfahrens- und Biotechnik; Fakultät 5: Informatik, Elektrotechnik und Informationstechnik; Fakultät 6: Luft- und Raumfahrttechnik und Geodäsie; Fakultät 7: Konstruktions-, Produktions- und Fahrzeugtechnik; Fakultät 8: Mathematik und Physik; Fakultät 9: PhilosophischHistorische Fakultät; Fakultät 10: Wirtschafts- und Sozialwissenschaften). 
aus mehreren Wellen basieren. Es wird, unter anderem, auf Unterschiede zwischen Bachelor- und Masterstudierenden eingegangen, wie auch auf den Einfluss von Eingangsvoraussetzungen auf die Wahrnehmung unterschiedlicher Aspekte im Verlauf des Semesters. Im vorliegenden Beitrag werden Ergebnisse auf der Ebene der Gesamtuniversität berichtet; fach- bzw. studiengangspezifische Analysen stehen bisher noch aus. Im Anschluss werden die Ergebnisse hinsichtlich hochschuldidaktischer Implikationen und strategischer Konsequenzen für den weiteren Digitalisierungsprozess und die Qualitätsentwicklung der Hochschullehre diskutiert.

\section{Empirische Ergebnisse}

\subsection{Erhebungswelle 1: Rahmenbedingungen und Eingangsvoraussetzungen}

Die technische Ausstattung der Studierenden lässt in der Gesamtschau keine gravierenden Engpässe ${ }^{4}$ erkennen (siehe Tab. 2). Internetzugang sowie Technik zur Dokumentenbearbeitung und für Videokonferenzen sind als Grundausstattung meist vorhanden - weniger als $5 \%$ berichten über Engpässe. Lediglich die Verfügbarkeit von Drucker/Kopierer und Scanner ist bei knapp $10 \%$ nicht gegeben, wird aber auch nur in begrenztem Rahmen für die Lehre benötigt.

\begin{tabular}{|lccc|}
\hline & $\begin{array}{c}\text { Zu Hause zur } \\
\text { Verfügung }\end{array}$ & $\begin{array}{c}\text { Zwingend } \\
\text { benötigt für } \\
\text { LV-Teilnahme }\end{array}$ & $\begin{array}{c}\text { Personen mit } \\
\text { Engpässen }\end{array}$ \\
\hline Internet/WLAN & $99.3 \%$ & $94.4 \%$ & $0.4 \%$ \\
Tablet oder Computer oder Laptop & $99.5 \%$ & $93.0 \%$ & $0.3 \%$ \\
Webcam/Kamera & $77.4 \%$ & $30.5 \%$ & $4.3 \%$ \\
Mikrofon/Headset & $77.6 \%$ & $52.9 \%$ & $5.3 \%$ \\
Smartphone & $97.0 \%$ & $16.8 \%$ & $0.4 \%$ \\
Drucker/Kopierer & $71.4 \%$ & $30.6 \%$ & $9.7 \%$ \\
Scanner & $61.2 \%$ & $23.7 \%$ & $8.2 \%$ \\
\hline
\end{tabular}

Tab. 2.: Technische Ausstattung der Studierenden.

Die Vertrautheit mit Computeranwendungen ist auf Seiten der Studierenden ebenso vorhanden - die Selbsteinschätzungen bewegen sich deutlich über dem theoretischen Skalenmittel und sind verhältnismässig heterogen (siehe Abb. 1). In der Gesamtstichprobe sind die befragten Studierenden am vertrautesten mit Internet und E-Mail. Signifikante, aber kleine Unterschiede zwischen Bachelor- und

4 Hierbei wird ein Engpass in dem Fall angenommen, wenn ein Studierender beispielsweise einen Scanner zwingend benötigt, um an digitalen Lehrveranstaltungen teilzunehmen, einen solchen jedoch nicht zu Hause zur Verfügung hat. 
Masterstudierenden lassen sich nur bei der Textverarbeitung (Hedges $g=-0.23$ ) und dem Umgang mit Computern im Allgemeinen (Hedges $g=-0.10$ ) zugunsten der Masterstudierenden feststellen. ${ }^{5}$

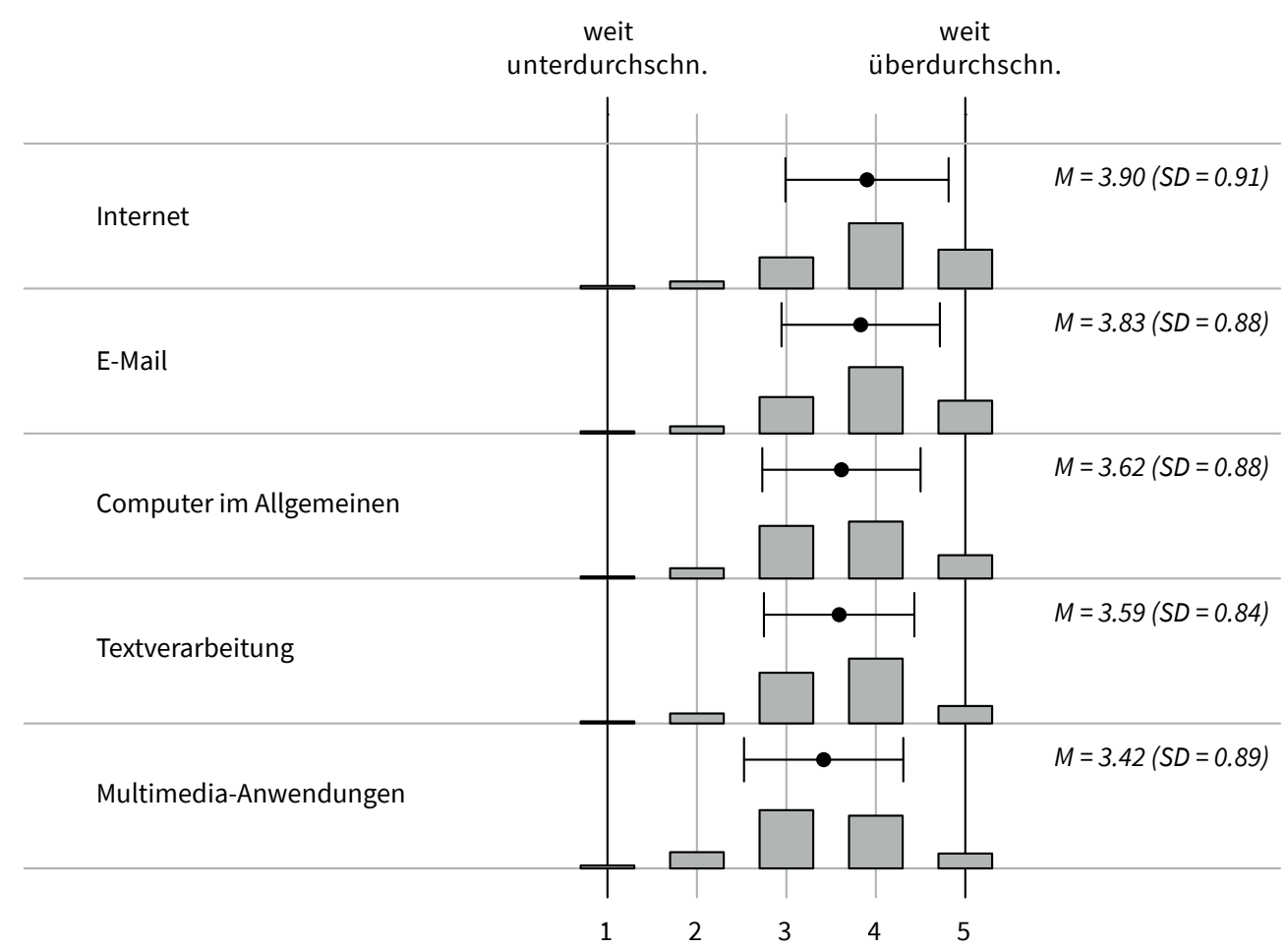

Abb. 1.: Vertrautheit mit Computeranwendungen.

Die emotional-motivationalen Eingangsvoraussetzungen der Studierenden zeichnen folgendes Bild: Während sich Bachelor- und Masterstudierende in ihrem Studieninteresse ${ }^{6}$ (Gesamtstichprobe und -skala $M=2.99$; $S D=0.47$ ) nicht signifikant unterscheiden, gibt es kleinere Unterschiede in den Dimensionen der Selbststeuerungsfähigkeit $^{7}$ (Gesamtstichprobe und -skala M $=3.33$; $S D=0.40$ ): So weisen Masterstudierende ein grösseres Mass an Selbstkontrolle und Zielverfolgung (Hedges $g=-0.12$ ) und Selbstmotivierung und Emotionskontrolle auf (Hedges $g=-0.21$ ) und prokrastinieren weniger als Bachelorstudierende (Hedges $g=0.10$ ). Bezüglich der IT-bezogenen Selbstwirksamkeit ${ }^{8}$ (Gesamtstichprobe $\mathrm{M}=3.99$; $\mathrm{SD}=0.95$ ) fühlen sich Masterstudierenden nach eigener Aussage besser für den Umgang mit Herausforderungen und

5 Im Rahmen des vorliegenden Beitrags wird im Falle von Mittelwertsunterschieden die Effektstärke Hedges g (vgl. z. B. Bortz und Schuster 2010, 127) berichtet. Diese berücksichtigt, anders als die Effektstärke Cohens d, die Grösse der zwei betrachteten Gruppen.

6 Skalenendpunkte: $1=$ geringes Interesse, $4=$ hohes Interesse.

7 Skalenendpunkte: 1 = geringe Selbststeuerungsfähigkeit; 5 = hohe Selbststeuerungsfähigkeit.

8 Skalenendpunkte: 1 = geringe Selbstwirksamkeitserwartung; $5=$ hohe Selbstwirksamkeitserwartung. 
Schwierigkeiten im Zusammenhang mit IT und digitalen Medien gewappnet (Hedges $\mathrm{g}=-0.15)$. Das beständige Interesse für das eigene Studium ${ }^{9}$ ist bei Masterstudierenden höher ausgeprägt als bei Bachelorstudierneden (Hedges $g=-0.17$ ), genauso wie die Beharrlichkeit im Rahmen des eigenen Studiums ${ }^{10}$ (Hedges $g=-0.24$ ).

Aufschlussreich war die Perspektive der Studierenden auf die Frage, welche Tools sich für die digitale Lehre eignen: Am besten sind aus Sicht der Studierenden (siehe Abb. 2) selbst erstellte Videos der Dozierenden ( $M=3.55)$, Screencasts oder Vorlesungsfolien im Videoformat $(M=3.48)$, weniger gut geeignet sind Texte zum reinen Selbststudium ( $M=2.59$ ) oder Foren auf ILIAS ( $M=2.63$ ). Dabei unterscheiden sich Bachelor- und Masterstudierende teilweise signifikant, wenn auch gering in ihren Einschätzungen: Masterstudierende halten etwa Videos (Hedges $g=-0.08$ ), besprochene Vorlesungsfolien (Hedges $g=-0.09$ ) und Texte (Hedges $g=-0.19$ ) zum Selbststudium für geeigneter als Bachelorstudierende.

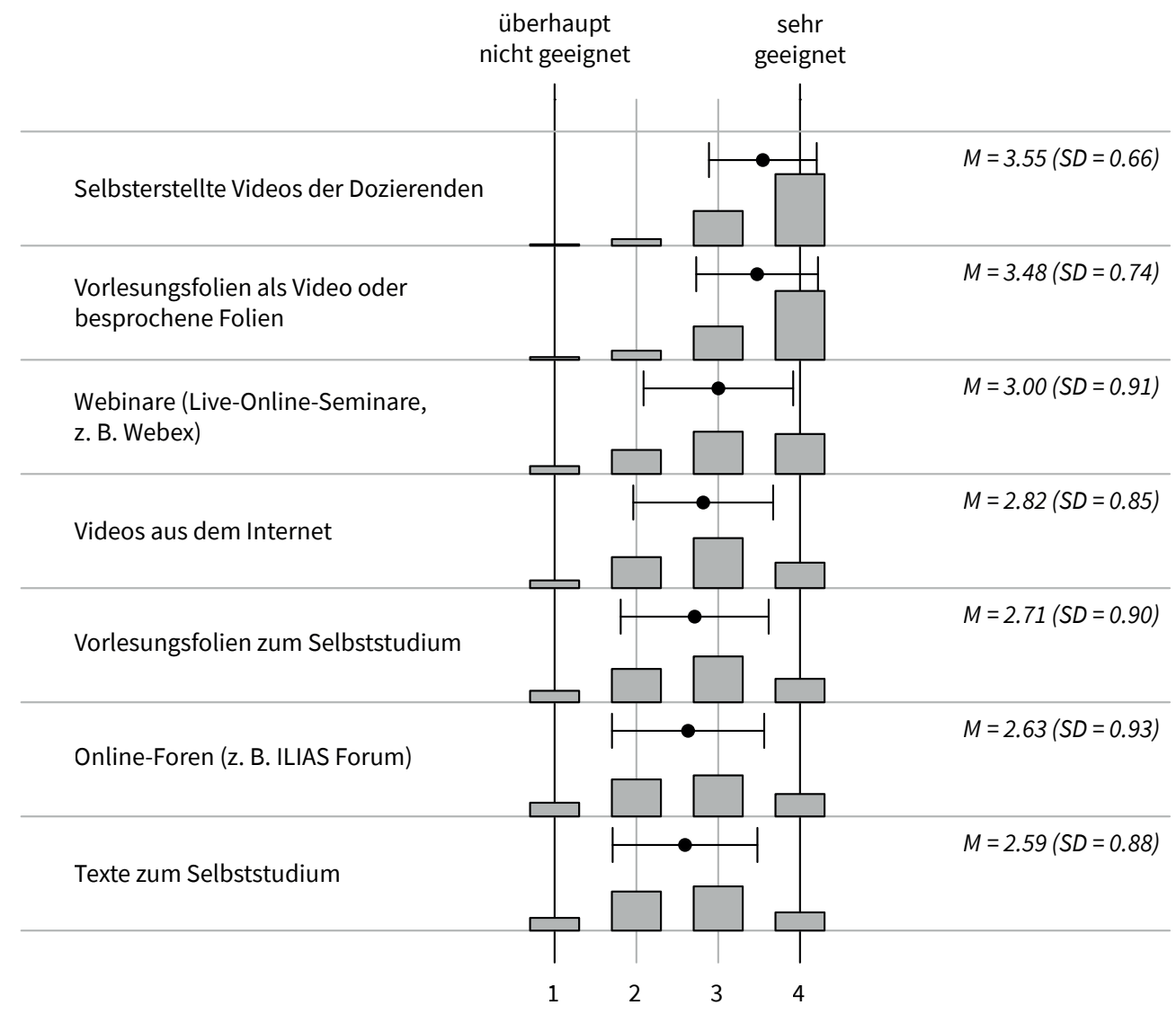

Abb. 2.: Eignung digitaler Lehr-Lern-Formate.

9 Skalenendpunkte: 1 = geringes beständiges Interesse; 5 = hohes beständiges Interesse.

10 Skalenendpunkte: 1 = geringe Beharrlichkeit; 5 = hohe Beharrlichkeit. 
Auch die Frage nach dem Erleben des häuslichen Lernens und spezifischen Störfaktoren (siehe Abb. 3) wurde von Bachelor- und Masterstudierenden unterschiedlich beantwortet: Insbesondere Bachelorstudierende berichten über mehr Probleme beim Selbststudium, die von Masterstudierenden signifikant angenehmer erlebt wird (Hedges $g=-0.09$ ). Masterstudierende berichten zudem über signifikant weniger Probleme mit dem Internet (Hedges $g=0.12$ ) und haben im allgemeinen weniger technische Probleme (Hedges $g=0.11$ ). Die Unterschiede weisen jedoch sehr geringe Effektstärken auf. Der Blick auf die Verteilung der Antworten auf die Items offenbart für die Gesamtstichprobe ein heterogenes Bild - lediglich mangelnde technische Kenntnisse scheinen in der befragten Stichprobe eine untergeordnete Rolle zu spielen, ansonsten gibt es offenbar sowohl Studierende, die Schwierigkeiten haben als auch jene, die keine haben und das Studieren von zuhause als angenehm empfinden.

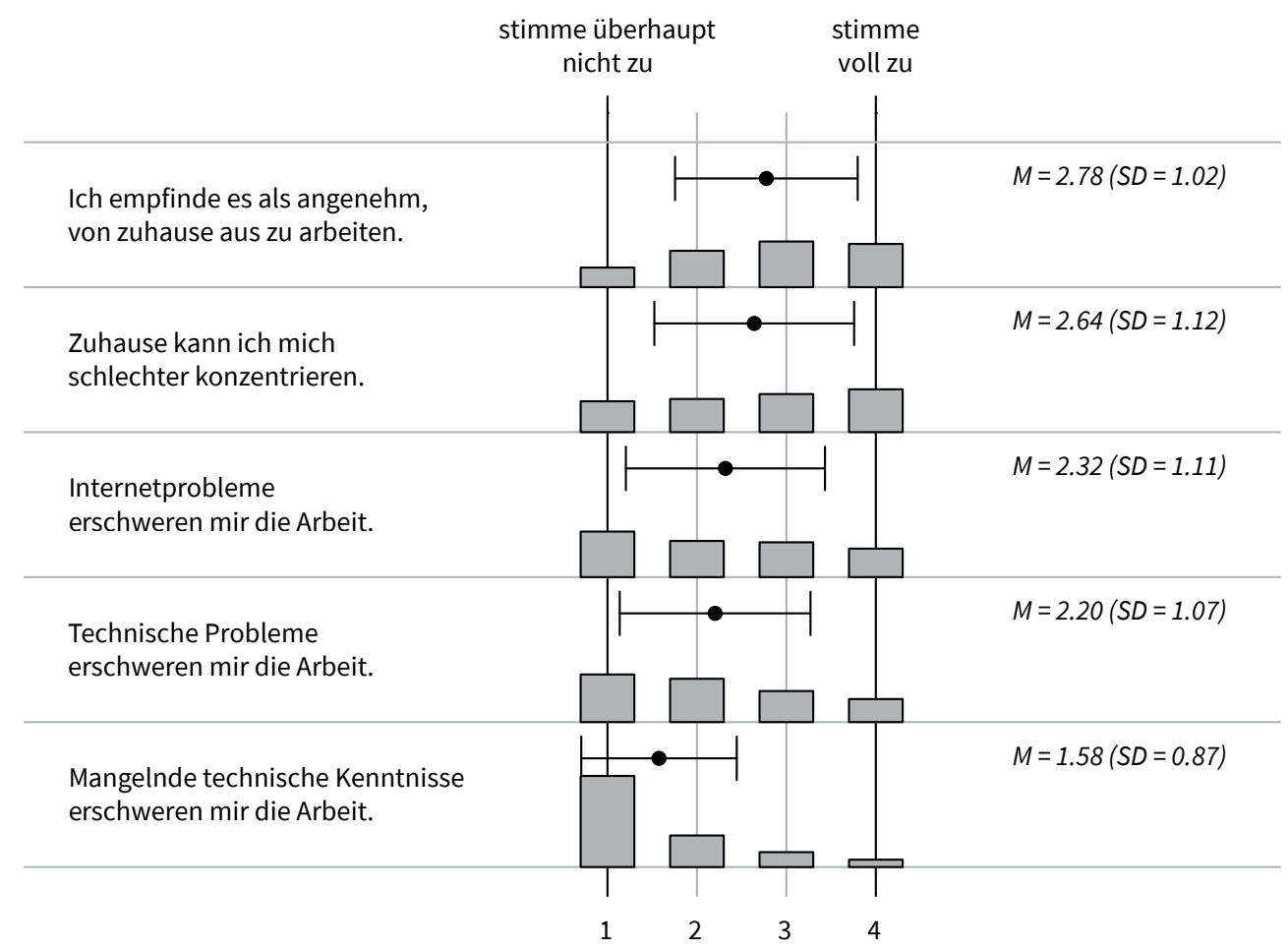

Abb. 3.: Erleben der Arbeit zuhause und Störfaktoren.

Der explorative Ansatz, das Erleben der Arbeit von zu Hause durch die Selbststeuerungsfähigkeit (Selbstkontrolle und Zielverfolgung, Selbstmotivierung und Emotionskontrolle sowie Prokrastination), die IT-bezogene Selbstwirksamkeit sowie das beständige Interesse sowie die Beharrlichkeit (GRIT-Skalen) regressionsanalytisch zu erklären, liefert folgende Ergebnisse: 
Auf die Aussage, inwiefern das Arbeiten von zu Hause als angenehm empfunden wird, haben die Prokrastination (standardisierter Regressionskoeffizient $\beta=-0.18$ ), die IT-bezogenen Selbstwirksamkeitserwartungen $(\beta=0.19)$ sowie die Beharrlichkeit $(\beta=-0.15)$ jeweils signifikante Einflüsse. Insgesamt wird hier aber lediglich eine Varianzaufklärung von $8 \%$ erreicht.

Die Varianz der Aussage «Zuhause kann ich mich schlechter konzentrieren» kann zu $15 \%$ erklärt werden durch Selbstkontrolle und Zielverfolgung ( $\beta=-0.10)$, Prokrastination (standardisierter Regressionskoeffizient $\beta=0.35$ ), IT-bezogenen Selbstwirksamkeitserwartungen ( $\beta=-0.11$ ) sowie die Beharrlichkeit $(\beta=0.13)$.

Die Varianz der Aussage «Internetprobleme erschweren mir die Arbeit» kann lediglich zu $5.5 \%$ aufgeklärt werden. Hier gibt es einen signifikanten Einfluss der ITbezogenen Selbstwirksamkeitserwartungen $(\beta=-0.23)$.

Die Varianz der Aussage «Technische Probleme erschweren mir die Arbeit» wird zu $14 \%$ aufgeklärt. Dabei haben folgende Variablen einen signifikanten Einfluss: Prokrastination $(\beta=0.08)$, beständiges Interesse $(\beta=-0.1)$ und Beharrlichkeit $(\beta=0.1)$ sowie IT-bezogene Selbstwirksamkeitserwartungen $(\beta=-0.35)$, die den grössten Einfluss haben.

«Mangelnde technische Kenntnisse erschweren mir die Arbeit» kann zu $40 \%$ erklärt werden durch Selbstkontrolle und Zielverfolgung $(\beta=-0.08)$, Selbstmotivierung und Emotionskontrolle $(\beta=0.10)$, Prokrastination $(\beta=0.09)$, die IT-bezogenen Selbstwirksamkeitserwartungen $(\beta=-0.61)$, das beständige Interesse $(\beta=-0.09)$ und die Beharrlichkeit $(\beta=0.11)$.

Insgesamt werden hier die Relevanz der Selbstwirksamkeitserwartungen hinsichtlich IT und digitalen Medien sowie die Rolle der Prokrastination im Rahmen der Selbststeuerungsfähigkeit von Studierenden ersichtlich.

\subsection{Erhebungswelle 2: Lernerfahrungen und Qualitätsurteile}

In der zweiten Erhebung gegen Ende der Vorlesungszeit wurden die Studierenden gefragt, wie sie mit verschiedenen Aspekten der aktuellen Studiensituation zurechtkamen. Für die Gesamtstichprobe zeigte sich (siehe Abb. 4), dass der fehlende direkte Kontakt zu den Kommilitoninnen und Kommilitonen, den Lehrverantwortlichen sowie die Schliessung der Bibliotheken und die Verschiebung von Prüfungen die grössten Schwierigkeiten mit sich brachten. Hier liegen die Durchschnittswerte über dem theoretischen Skalenmittel. Weniger relevant scheint die Schliessung von PCPools oder Cafeterien und Mensen gewesen zu sein. Signifikante, wenn auch kleine Unterschiede zeigen sich wiederum zwischen Bachelor- und Masterstudierenden letztere kommen besser mit der Vorbereitung auf Prüfungen (Hedges $g=0.17$ ), der digitalen Lehre im Allgemeinen (Hedges $g=0.20$ ) und den bestehenden Unterschieden zwischen Studierenden bei der Gestaltung der digitalen Lehre (Hedges $g=0.12$ ) 
zurecht. Bezüglich des Zurechtkommens mit der digitalen Lehre im Allgemeinen wurden zusätzlich signifikante Unterschiede zwischen unerfahrenen Studierenden (Bachelor im 1./2. Fachsemester) und Studierenden höherer Fachsemester identifiziert (Hedges $g=0.25$ ), letztere tun sich deutlich leichter.

Zusätzlich zur Interpretation der Durchschnittswerte kann die Betrachtung der Streuungen erkenntnisbringend sein: In Abb. 4 wird ersichtlich, dass die Schliessung der Bibliotheken, die Schliessung der Lernräume sowie die Schliessung der PC-Pools grössere Standardabweichungen aufweisen als die anderen Aspekte. Dies bedeutet, dass es hier scheinbar grössere Unterschiede zwischen den Studierenden gibt. Potentielle Ursachen für diese Unterschiede wurden nicht erfragt. Denkbar sind möglicherweise die Wohnsituation der Studierenden oder der Studiengang.

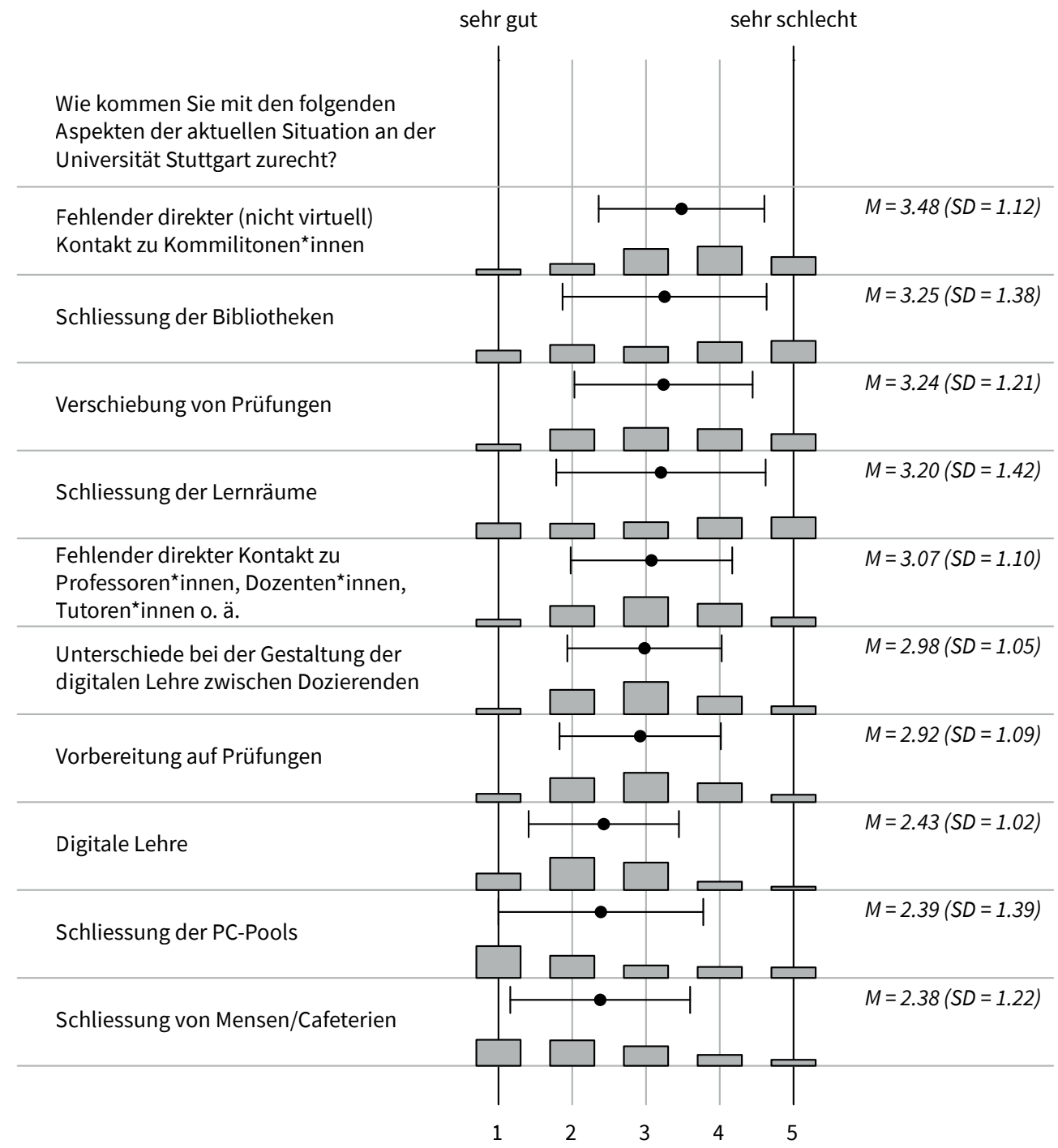

Abb. 4.: Zurechtkommen in der aktuellen Situation. 
Weiterhin wurden die Teilnehmenden im zweiten Erhebungszeitpunkt nach ihren Erfahrungen mit dem digitalen Studium befragt (siehe Abb. 5). Im Mittel werden zwar die zeitliche Flexibilität und ein zunehmend reibungsloses Funktionieren der digitalen Lehrformate geschätzt, aber bei der Frage nach der Effizienz des digitalen Studiums im Vergleich zum Präsenzstudium und der Möglichkeit der Klärung von Fragen gehen die Meinungen auseinander. Auch hier sind in der Regel Masterstudierende diejenigen, die das digitale Studium etwas besser bewerten.

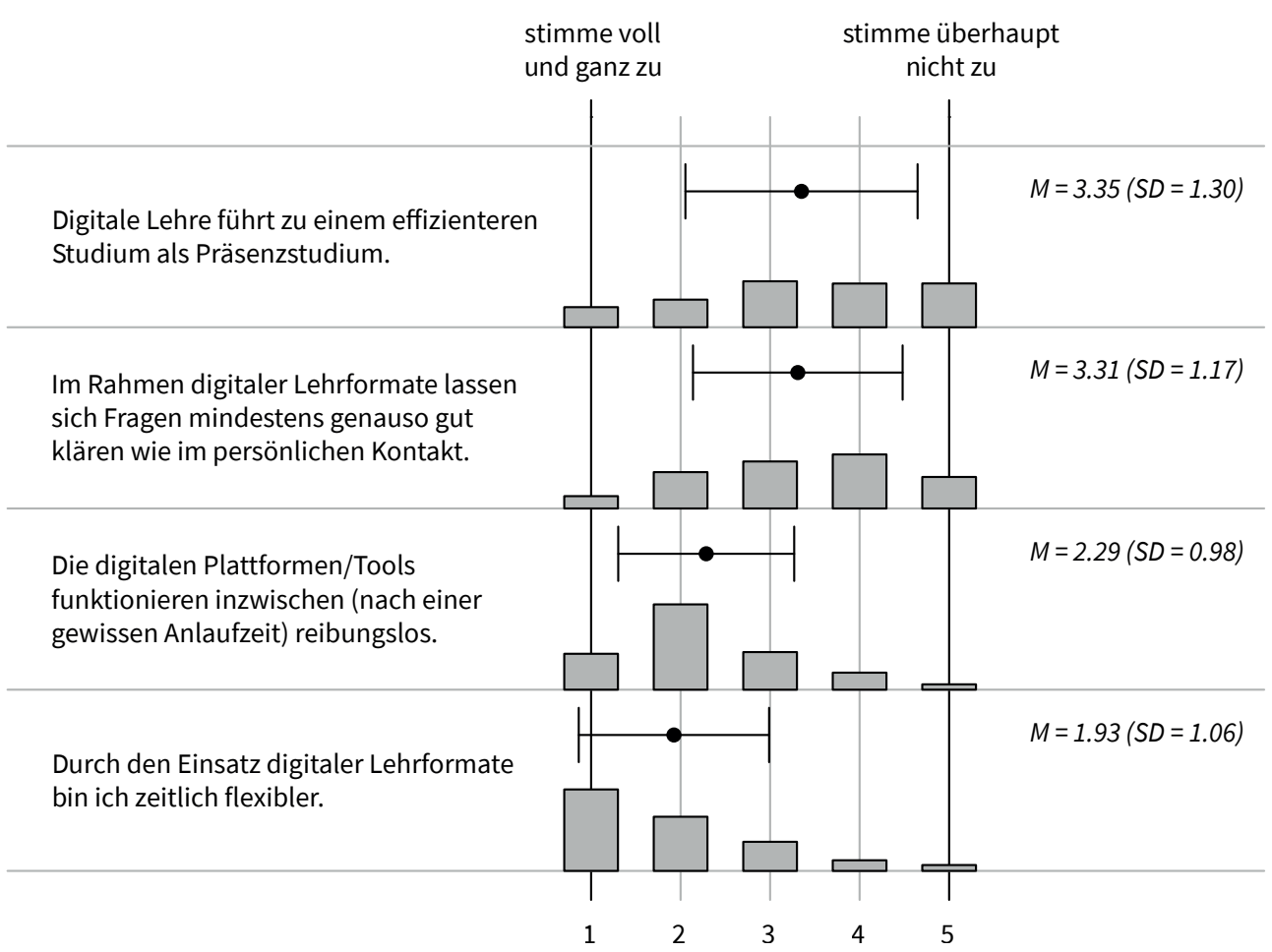

Abb. 5.: Erfahrungen mit digitaler Lehre und Studium.

Die Frage nach der Zufriedenheit mit der Gestaltungsqualität unterschiedlicher Formate der digitalen Lehre beantworten die Studierenden im Mittel recht positiv (siehe Abb. 6). Die Arbeit mit der Lernplattform ILIAS scheint verhältnismässig zufriedenzustellen, und auch die Qualität der Lernmaterialien wird als gut bewertet. Masterstudierende bewerten die Qualität der Audiodateien deutlich besser als ihre Kommilitoninnen und Kommilitonen in Bachelorstudiengängen (Hedges $g=0.23$ ). 


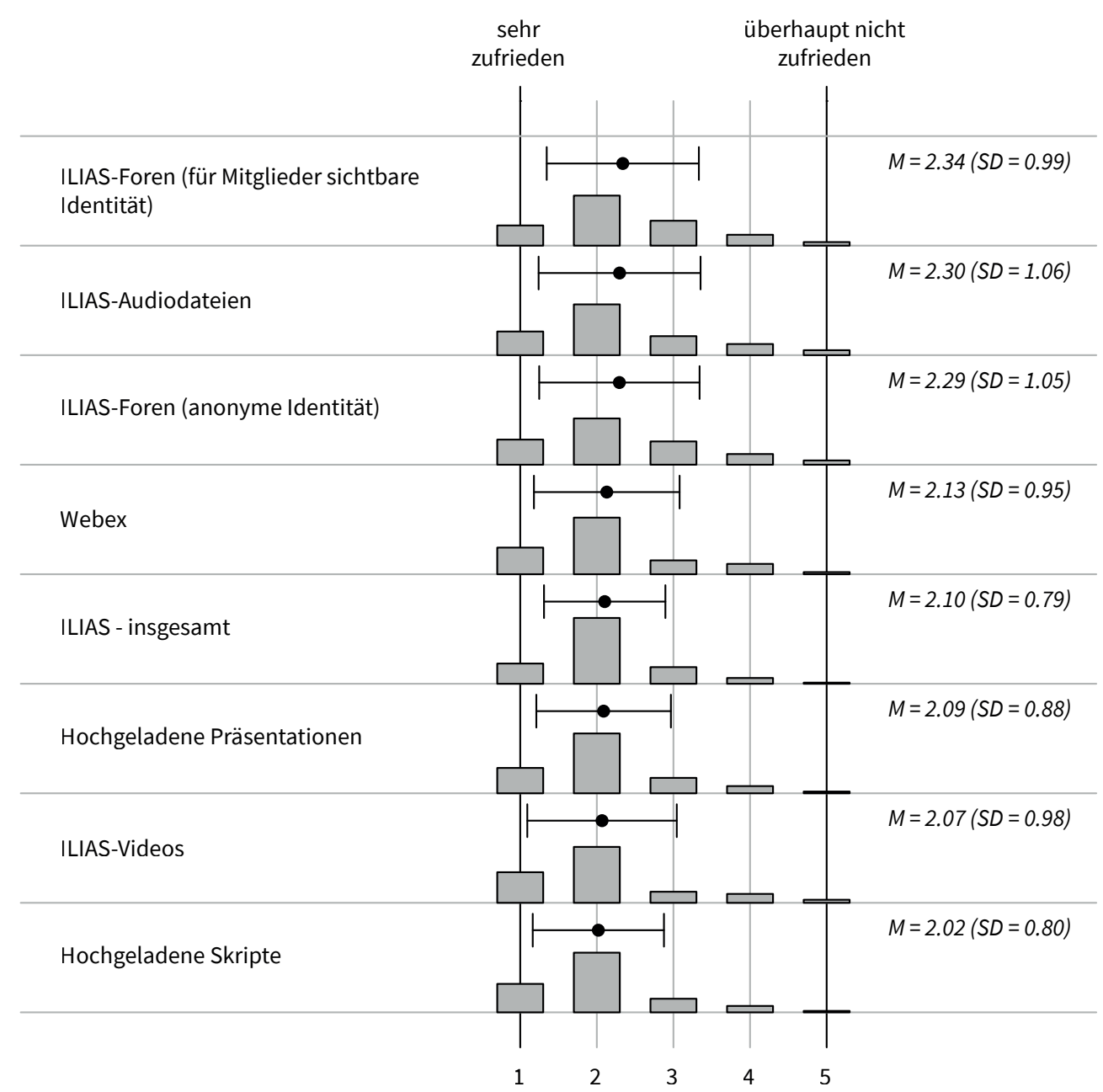

Abb. 6.: Zufriedenheit mit der Gestaltungsqualität digitaler Lehre.

Nichtsdestoweniger haben gut $38 \%$ der Studierenden bereits im zweiten Messzeitpunkt den Eindruck, dass sie ihr Semester nicht wie geplant abschliessen können. Die Gründe dafür sind im Wesentlichen mit der Corona-Pandemie assoziiert und werden insbesondere in der höheren Arbeitsbelastung, einem unzureichenden Arbeitsklima, einer verbesserungswürdigen Prüfungsorganisation sowie der fehlenden Interaktion mit den Kommilitoninnen und Kommilitonen gesehen (siehe Tab. 3). 


\begin{tabular}{|c|c|c|c|c|c|}
\hline $\begin{array}{l}\text { Falls Sie Sie Ihr Studienpro- } \\
\text { gramm im derzeit laufenden } \\
\text { Semester nicht wie geplant } \\
\text { erfüllen können: } \\
\text { Was sind die Hauptgründe } \\
\text { dafür? }\end{array}$ & Grund & Bachelor & Master & $\begin{array}{l}\text { Phi-Koeff: } \\
\text { Korr. zw. } \\
\text { Unter- } \\
\text { stüztzung } \\
\text { und Ab- } \\
\text { schlussart }\end{array}$ & p-Wert \\
\hline $\begin{array}{l}\text { Höhere Arbeitsbelastung/ } \\
\text { Arbeitsaufwand }\end{array}$ & $33.2 \%$ & $36.7 \%$ & $28.0 \%$ & & n.s. \\
\hline Schlechte Prüfungsorganisation & $29.9 \%$ & $29.3 \%$ & $31.3 \%$ & & n.s. \\
\hline $\begin{array}{l}\text { Fehlende Gruppenarbeit/ } \\
\text { Kommunikation mit } \\
\text { Kommilitonen*innen }\end{array}$ & $29.6 \%$ & $32.2 \%$ & $24.6 \%$ & -0.079 & 0.03 \\
\hline $\begin{array}{l}\text { Unzureichendes Arbeitsklima } \\
\text { (Ruhe, Räumlichkeit) }\end{array}$ & $25.3 \%$ & $26.4 \%$ & $22.8 \%$ & & n.s. \\
\hline $\begin{array}{l}\text { Wichtiger Präsenzteil/Pra- } \\
\text { xisteil des Studiums nicht } \\
\text { wahrnehmbar }\end{array}$ & $20.9 \%$ & $20.6 \%$ & $22.4 \%$ & & n.s. \\
\hline $\begin{array}{l}\text { Emotionale Belastung durch } \\
\text { die Corona-Situation }\end{array}$ & $18.6 \%$ & $19.4 \%$ & $17.2 \%$ & & n.s. \\
\hline $\begin{array}{l}\text { Schliessung der Bibliotheken } \\
\text { bzw. eingeschränkte Öffnungs- } \\
\text { zeiten von Bibliotheken }\end{array}$ & $17.2 \%$ & $18.1 \%$ & $14.9 \%$ & & n.s. \\
\hline $\begin{array}{l}\text { Unzureichende inhaltli- } \\
\text { che Qualität der digitalen } \\
\text { Lehrveranstaltungen }\end{array}$ & $15.9 \%$ & $18.6 \%$ & $11.2 \%$ & -0.096 & 0.008 \\
\hline $\begin{array}{l}\text { Überschneidung von } \\
\text { Abgabefristen }\end{array}$ & $10.4 \%$ & $9.5 \%$ & $12.3 \%$ & & \\
\hline $\begin{array}{l}\text { Zu geringes digitales } \\
\text { Lehrangebot }\end{array}$ & $8.3 \%$ & $6.4 \%$ & $11.6 \%$ & 0.09 & 0.013 \\
\hline $\begin{array}{l}\text { Unzureichende Möglichkeit, } \\
\text { Prüfungen abzulegen }\end{array}$ & $8.2 \%$ & $7.2 \%$ & $10.4 \%$ & & n.s. \\
\hline Finanzielle Engpässe & $7.4 \%$ & $6.0 \%$ & $10.1 \%$ & -0.088 & 0.015 \\
\hline $\begin{array}{l}\text { Unzureichende technische Qua- } \\
\text { lität der Lehrveranstaltungen }\end{array}$ & $5.0 \%$ & $4.5 \%$ & $5.2 \%$ & & \\
\hline $\begin{array}{l}\text { Fehlende Hilfs- und } \\
\text { Unterstützungsangebote }\end{array}$ & $4.6 \%$ & $5.8 \%$ & $2.6 \%$ & -0.072 & 0.049 \\
\hline $\begin{array}{l}\text { Unzureichende techni- } \\
\text { sche Ausstattung zuhause } \\
\text { (z. B. Internetzugang) }\end{array}$ & $4.0 \%$ & $3.1 \%$ & $6.0 \%$ & -0.041 & 0.057 \\
\hline $\begin{array}{l}\text { Ehrenamtliche Tätigkeiten in } \\
\text { Zusammenhang mit Corona }\end{array}$ & $1.8 \%$ & $2.1 \%$ & $1.1 \%$ & & n.s. \\
\hline Fehlende Kinderbetreuung & $0.7 \%$ & $0.4 \%$ & $1.1 \%$ & & n.s. \\
\hline
\end{tabular}

Tab. 3.: Gründe für geringere Semesterleistung (Mehrfachantworten möglich). 
Mehr Unterstützung wünschen sich die Bachelorstudierenden insbesondere bei der Vorbereitung auf Prüfungen, bei der Lernberatung und Tagesstrukturierung (s. Tab. 3). In der Gesamtschau zeichnet sich überdies Unterstützungsbedarf bei der Organisation Lerngruppen ab. Ein Drittel aller Befragten wünscht sich hierbei umfassendere Unterstützung.

\begin{tabular}{|c|c|c|c|c|c|}
\hline $\begin{array}{l}\text { In welchen der folgenden Situ- } \\
\text { ationen hätten Sie sich (mehr) } \\
\text { Unterstützung seitens der Uni } \\
\text { gewünscht? }\end{array}$ & $\begin{array}{l}\text { (mehr) } \\
\text { Unterstüt- } \\
\text { zung }\end{array}$ & Bachelor & Master & $\begin{array}{l}\text { Phi-Koeff: } \\
\text { Korr. zw. } \\
\text { Unter- } \\
\text { stüztzung } \\
\text { und Ab- } \\
\text { schlussart }\end{array}$ & p-Wert \\
\hline Vorbereitung auf Prüfungen & $55.9 \%$ & $60.8 \%$ & $48.4 \%$ & -0.12 & $<0.001$ \\
\hline Tagesstrukturierung & $32.1 \%$ & $36.4 \%$ & $24.5 \%$ & -0.123 & $<0.001$ \\
\hline Lerngruppe finden & $29.3 \%$ & $29.0 \%$ & $29.6 \%$ & & n.s. \\
\hline Einführung in Webex & $19.1 \%$ & $20.3 \%$ & $16.7 \%$ & -0.044 & 0.046 \\
\hline Lernberatung & $18.2 \%$ & $20.6 \%$ & $14.5 \%$ & -0.076 & 0.001 \\
\hline Psychologische Beratung & $17.9 \%$ & $18.1 \%$ & $17.5 \%$ & & n.s. \\
\hline $\begin{array}{l}\text { Allgemeine Unterstützung zu } \\
\text { technischen Angelegenheiten }\end{array}$ & $17.7 \%$ & $18.1 \%$ & $16.4 \%$ & & n.s. \\
\hline Stundenplan erstellen & $16.3 \%$ & $17.1 \%$ & $15.0 \%$ & & n.s. \\
\hline Einführung in ILIAS & $7.2 \%$ & $7.5 \%$ & $6.4 \%$ & & n.s. \\
\hline
\end{tabular}

Tab. 4.: Unterstützungsbedarf (Mehrfachantworten möglich).

Schliesslich wurden die Studierenden zum zweiten Messzeitpunkt nach ihrem subjektiven Wohlbefinden hinsichtlich verschiedener Aspekte befragt (s. Abb. 7). Besonders die fehlenden sozialen Kontakte verursachen dabei ein hohes Belastungsempfinden. Darüber hinaus kämpfen die Studierenden mit fehlender Motivation, mangelnder Selbststrukturierung und allgemeinen Anpassungsschwierigkeiten an die neuen Lehrformate. 


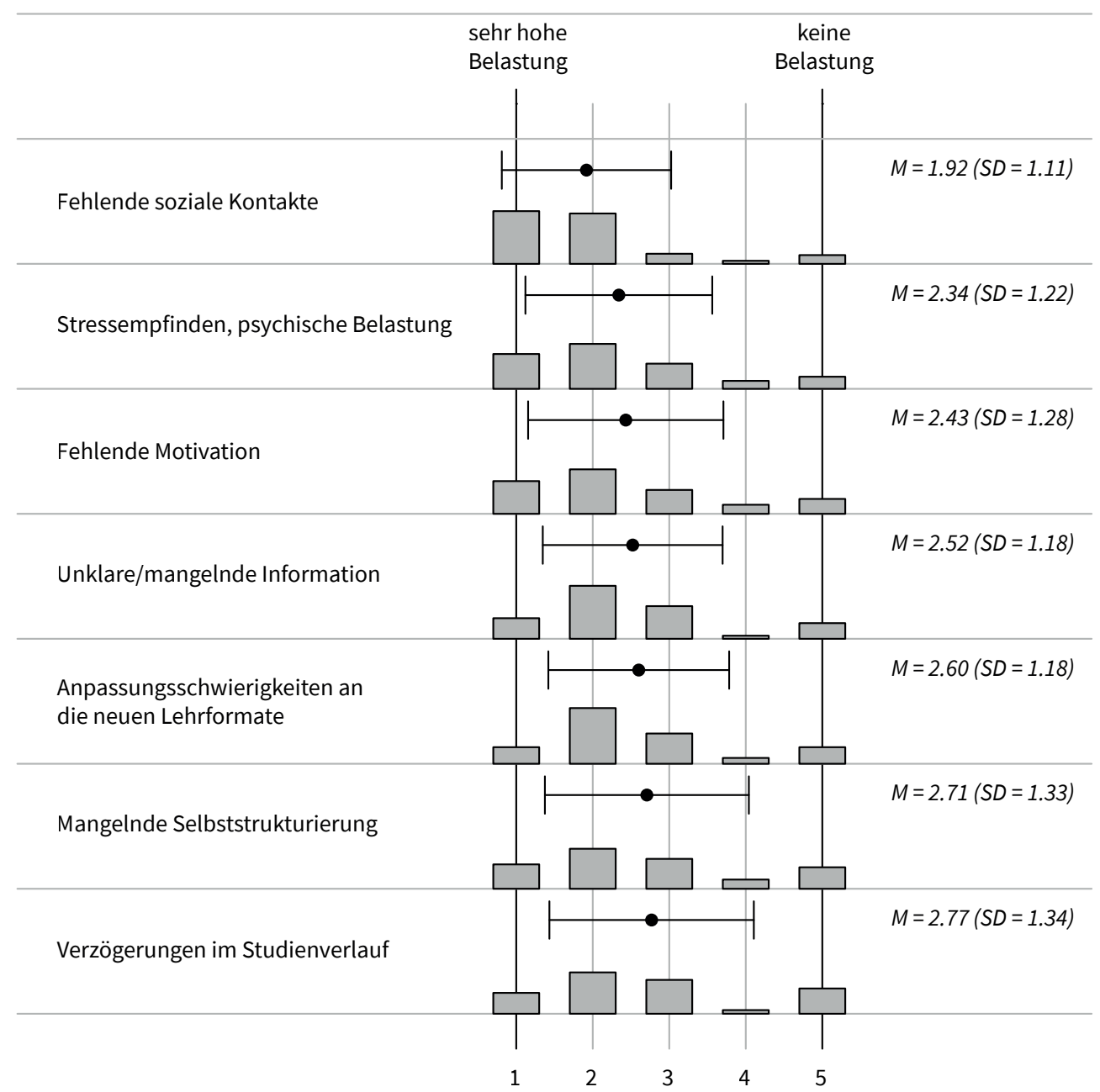

Abb. 7.: Wohlbefinden der Studierenden.

Kleinere Unterschiede zugunsten der Masterstudierenden zeigen sich im allgemeinen Stressempfindens (Hedges $g=-0.10$ ), der fehlenden Motivation (Hedges $\mathrm{g}=-0.14$ ) bezüglich Anpassungsschwierigkeiten (Hedges $\mathrm{g}=-0.11$ ).

\subsection{Erhebungswelle 3: Subjektive Erfolgsbilanz und Folgeerwartungen}

Nach Beendigung der Prüfungsphase wurden die Studierenden im Oktober 2020 nach der Anzahl der erbrachten Prüfungen gefragt. Es zeigt sich, dass knapp die Hälfte der Teilnehmenden weniger Prüfungen absolvieren konnte als ursprünglich geplant, während gut $47.1 \%$ ihr geplantes Pensum erfüllen konnten. Der Grossteil der Befragten hat dabei eine oder zwei Prüfungen weniger erbracht als ursprünglich geplant. 


\begin{tabular}{|c|c|c|c|c|c|c|c|c|c|c|c|}
\hline \multicolumn{12}{|c|}{ Prüfungen im SoSe2020 tatsächlich erbracht } \\
\hline & & 0 & 1 & 2 & 3 & 4 & 5 & 6 & 7 & $\begin{array}{l}80 . \\
\text { mehr }\end{array}$ & Gesamt \\
\hline \multirow{8}{*}{$\begin{array}{l}\text { Prüfungen } \\
\text { für SoSe } \\
2020 \\
\text { geplant }\end{array}$} & 0 & 82 & 2 & 3 & 4 & 1 & 0 & 0 & 1 & 0 & 93 \\
\hline & 1 & 10 & 56 & 2 & 1 & 0 & 0 & 0 & 0 & 0 & 69 \\
\hline & 2 & 6 & 19 & 101 & 8 & 2 & 1 & 0 & 0 & 0 & 137 \\
\hline & 3 & 11 & 25 & 80 & 179 & 5 & 2 & 1 & 0 & 0 & 303 \\
\hline & 4 & 7 & 21 & 41 & 127 & 170 & 6 & 4 & 1 & 1 & 378 \\
\hline & 5 & 6 & 11 & 24 & 80 & 133 & 179 & 10 & 2 & 1 & 446 \\
\hline & 6 & 2 & 2 & 13 & 27 & 63 & 93 & 112 & 5 & 4 & 321 \\
\hline & 7 & 3 & 0 & 6 & 7 & 19 & 46 & 52 & 37 & 2 & 172 \\
\hline \multicolumn{2}{|c|}{8 o. mehr } & 0 & 0 & 0 & 3 & 7 & 19 & 24 & 28 & 48 & 129 \\
\hline \multicolumn{2}{|l|}{ Gesamt } & 127 & 136 & 270 & 436 & 400 & 346 & 203 & 74 & 56 & 2048 \\
\hline
\end{tabular}

Tab. 5.: Prüfungspensum. Anmerkungen: Diagonale $=$ die geplanten Prüfungen erbracht: $\mathrm{N} 1=964(47.07 \%)$. Unterhalb der Diagonale = weniger Prüfungen erbracht als geplant: N2 $=1015(49.56 \%)$. Oberhalb der Diagonalen = mehr Prüfungen erbracht als geplant: N3 $=69(3.37 \%)$.

Die vordringlichen Gründe für das geringere Prüfungspensum, die mit der pandemiebedingten Fernlehre assoziiert sind, sehen Studierende etwa in dem fehlenden Lernen mit Kommilitoninnen und Kommilitonen (25.0\%), zu geringer Motivation $(18.7 \%)$ oder dem Fehlen eines ruhigen Lernortes (12.7 \%). Die geringere Verfügbarkeit von Literatur und defizitäre technische Ausrüstungen spielen auf die gesamte Studierendenschaft bezogen eine untergeordnete Rolle (s. Tab. 4). Signifikante Unterschiede zwischen Bachelor- und Masterstudierenden zeigen sich nur bei einigen Items: So bewerten Bachelorstudierende die eigene Prüfungsvorbereitung als weniger gut, wünschen sich eine bessere Prüfungs- und Veranstaltungsorganisation der Universität und mehr verfügbare Fachliteratur.

\begin{tabular}{|lccccc|}
\hline $\begin{array}{l}\text { Falls Sie in diesem Semester } \\
\text { weniger Prüfungsleistungen } \\
\text { erbracht haben als geplant: } \\
\text { Was waren die Hauptgründe } \\
\text { dafür? }\end{array}$ & Grund & Bachelor & Master & $\begin{array}{c}\text { Phi-Koeff: } \\
\text { Korr. zw. } \\
\text { Unter- } \\
\text { stüztzung } \\
\text { und Ab- }\end{array}$ & p-Wert \\
schlussart & \\
\hline $\begin{array}{l}\text { Das Lernen mit Kommilitonin- } \\
\text { nen/Kommilitonen hat gefehlt }\end{array}$ & $40.4 \%$ & $44.4 \%$ & $33.2 \%$ & & n.s. \\
\hline $\begin{array}{l}\text { Zeitliche Überschneidung/en } \\
\text { Zu viel Lernstoff }\end{array}$ & $37.2 \%$ & $38.5 \%$ & $35.4 \%$ & n.s. \\
$\begin{array}{l}\text { Zu geringe Motivation zum } \\
\text { Lernen/oder Abschliessen einer } \\
\text { Prüfungsleistung }\end{array}$ & $31.1 \%$ & $32.8 \%$ & $28.6 \%$ & & n.s. \\
$\begin{array}{l}\text { Unzureichende Prüfungsvorbe- } \\
\text { reitung meinerseits }\end{array}$ & $29.8 \%$ & $33.3 \%$ & $23.5 \%$ & -0.107 & 0.001 \\
\hline
\end{tabular}




\begin{tabular}{|c|c|c|c|c|c|}
\hline $\begin{array}{l}\text { Falls Sie in diesem Semester } \\
\text { weniger Prüfungsleistungen } \\
\text { erbracht haben als geplant: } \\
\text { Was waren die Hauptgründe } \\
\text { dafür? }\end{array}$ & Grund & Bachelor & Master & $\begin{array}{l}\text { Phi-Koeff: } \\
\text { Korr. zw. } \\
\text { Unter- } \\
\text { stüztzung } \\
\text { und Ab- } \\
\text { schlussart }\end{array}$ & p-Wert \\
\hline $\begin{array}{l}\text { Ruhiger Lernort hat gefehlt } \\
\text { und/oder war nicht lang genug } \\
\text { geöffnet }\end{array}$ & $25.6 \%$ & $26.2 \%$ & $23.5 \%$ & & n.s. \\
\hline $\begin{array}{l}\text { Inhalte der Veranstaltung } \\
\text { wurden nicht gut vermittelt }\end{array}$ & $21.6 \%$ & $22.6 \%$ & $19.5 \%$ & & n.s. \\
\hline $\begin{array}{l}\text { Insgesamt schlechte Prüfungs- } \\
\text { organisation seitens der Uni }\end{array}$ & $18.9 \%$ & $21.0 \%$ & $15.7 \%$ & -0.066 & 0.038 \\
\hline $\begin{array}{l}\text { Zu schwieriges Thema/ } \\
\text { Veranstaltung }\end{array}$ & $17.0 \%$ & $18.3 \%$ & $15.1 \%$ & & n.s. \\
\hline Persönliche Gründe/Probleme & $13.4 \%$ & $14.2 \%$ & $11.9 \%$ & & n.s. \\
\hline $\begin{array}{l}\text { Unzureichende Prüfungsvorbe- } \\
\text { reitung seitens des Dozenten }\end{array}$ & $11.3 \%$ & $10.3 \%$ & $12.7 \%$ & & n.s. \\
\hline $\begin{array}{l}\text { Veranstaltung/en wurde/n } \\
\text { abgesagt }\end{array}$ & $10.7 \%$ & $8.2 \%$ & $14.9 \%$ & 0.104 & 0.001 \\
\hline $\begin{array}{l}\text { Zu wenig Literatur, da Biblio- } \\
\text { theken geschlossen waren oder } \\
\text { zu kurze Öffnungszeiten hatten }\end{array}$ & $9.9 \%$ & $11.7 \%$ & $5.7 \%$ & -0.099 & 0.002 \\
\hline $\begin{array}{l}\text { Ich konnte krankheitsbedingt } \\
\text { nicht teilnehmen }\end{array}$ & $4.9 \%$ & $5.4 \%$ & $4.3 \%$ & & n.s. \\
\hline $\begin{array}{l}\text { Technische Ausrüstung hat } \\
\text { gefehlt/zu schlecht (z. B. PC, } \\
\text { Internetverbindung) }\end{array}$ & $5.1 \%$ & $5.7 \%$ & $3.8 \%$ & & n.s. \\
\hline
\end{tabular}

Tab. 6.: Gründe für geringeres Prüfungspensum (Mehrfachantworten möglich).

Von der Anzahl der geplanten Prüfungen wurde die Anzahl der tatsächlich erbrachten Prüfungen abgezogen, woraus die Differenzvariable aus Abbildung 8 resultiert. Ein Differenzwert von Null bedeutet, dass genau so viele Prüfungen geschrieben wurden wie geplant, eine positive Zahl bedeutet, dass weniger Prüfungen als geplant erbracht wurden. Einen signifikanten Einfluss auf diese Differenzvariable haben folgende Variablen: Prokrastination $(\beta=0.13)$, das beständige Interesse für das eigene Studium ( $\beta=-0.08$ ) sowie die Beharrlichkeit im Rahmen des eigenen Studiums $(\beta=-0.10)$. Die Varianzaufklärung erreicht $6.5 \%$. 


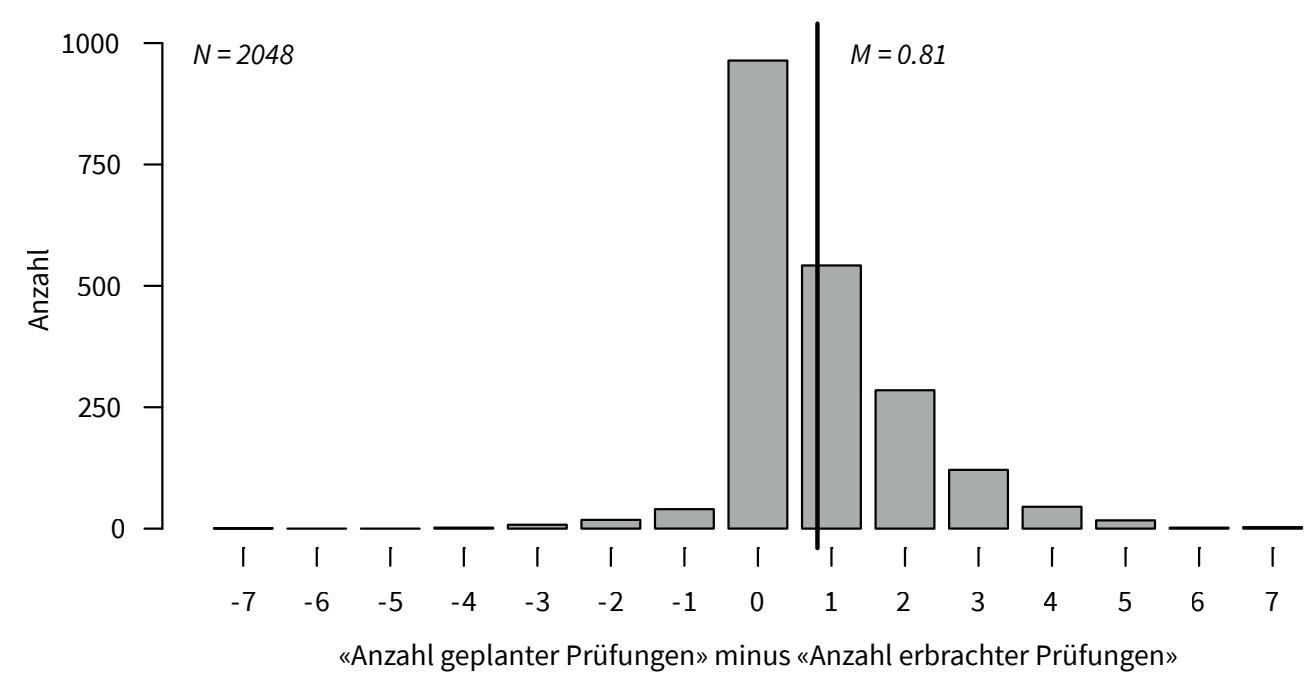

Abb. 8.: Histogramm: Anzahl geplanter Prüfungen minus Anzahl erbrachter Prüfungen.

Zudem wurden die Studierenden nach ihrer Einschätzung bezüglich der Prüfungsanforderungen im Vergleich zu der Zeit vor der Covid-19-Pandemie gefragt. Hier zeichnet sich ein heterogenes Bild ab (Abb. 9), im Mittel wird zwar nicht davon ausgegangen, dass die Prüfungsanforderungen gestiegen sind, während allerdings etwas mehr Zeit für die Vorbereitung benötigt wurde. Bachelorstudierende unterscheiden sich hier durchgehend signifikant von Masterstudierenden, wenn auch die Unterschiede nur geringe Effektstärken aufweisen - Masterstudierende bewerten dies weniger gravierend. Dabei scheinen persönliche Faktoren, die in der ersten Erhebungswelle ermittelt wurden, lediglich einen geringen Einfluss zu haben - beispielsweise können im Längsschnitt lediglich $7 \%$ der Varianz der wahrgenommenen erhöhten Zeit für die Prüfungsvorbereitung über die erhobenen Traits aufgeklärt werden: Je mehr man prokrastiniert $(\beta=-0.14)$, je geringer die IT-bezogenen Selbstwirksamkeitserwartungen sind $(\beta=0.08)$, je geringer das beständige Interesse für das eigene Studium $(\beta=0.17$ ) ist bzw. je höher die Beharrlichkeit im Rahmen des eigenen Studiums ( $\beta=-0.29$ ) ist, desto eher gibt man an, dass man mehr Zeit für die Prüfungsvorbereitung benötigt als sonst. 


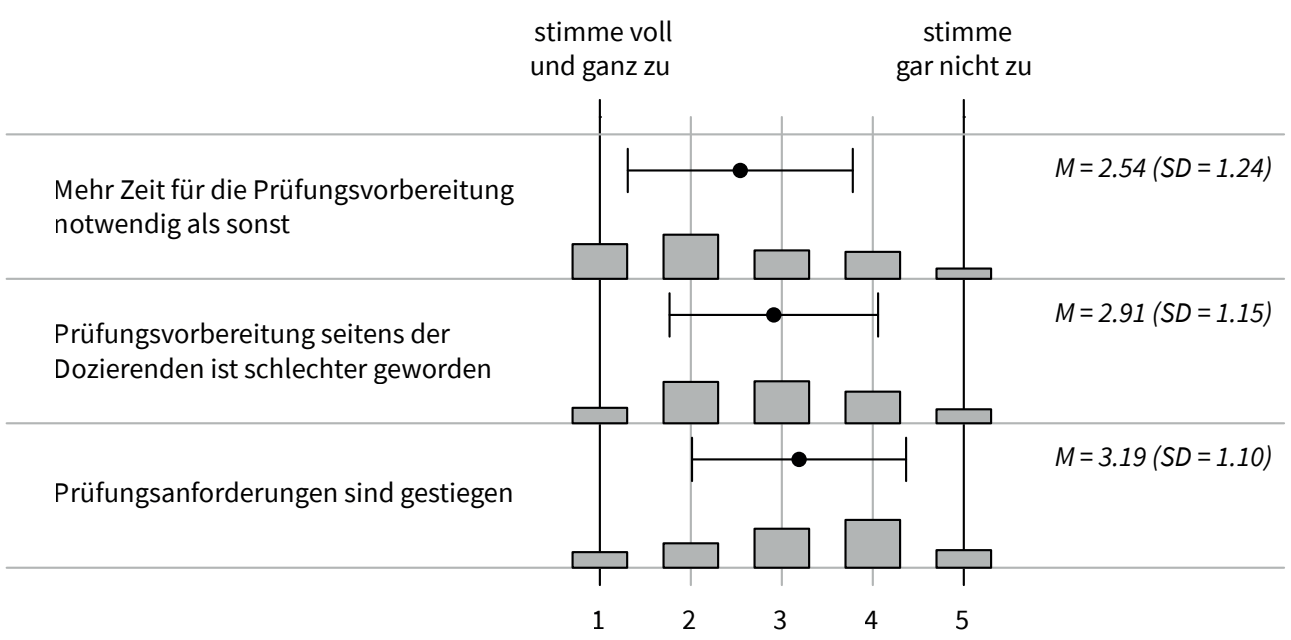

Abb. 9.: Prüfungsanforderungen im Vergleich.

Insgesamt scheinen Studierende zufrieden mit der Erreichbarkeit der Lehrenden und der Betreuung durch diese. Hinsichtlich des erreichten Wissens und Könnens ist das Stimmungsbild etwas differenzierter (Abb. 10). Dabei kann die Varianz der Zufriedenheit mit dem erreichten Wissen und Können zu 13 \% durch persönlichkeitsbezogene Faktoren aus der ersten Welle aufgeklärt werden: Die Zufriedenheit ist umso höher, je weniger prokrastiniert wird $(\beta=-0.20)$, je höher die IT-bezogenen Selbstwirksamkeitserwartungen sind $(\beta=0.13)$, je geringer die Beharrlichkeit ist $(\beta=-0.11$ ), je höher das beständige Interesse ist $(\beta=0.06)$, je höher die persönlichen wertbezogenen Valenzen für das Studium sind $(\beta=0.24)$ bzw. je geringer der intrinsische Charakter des Studiums ist $(\beta=-0.16)$.

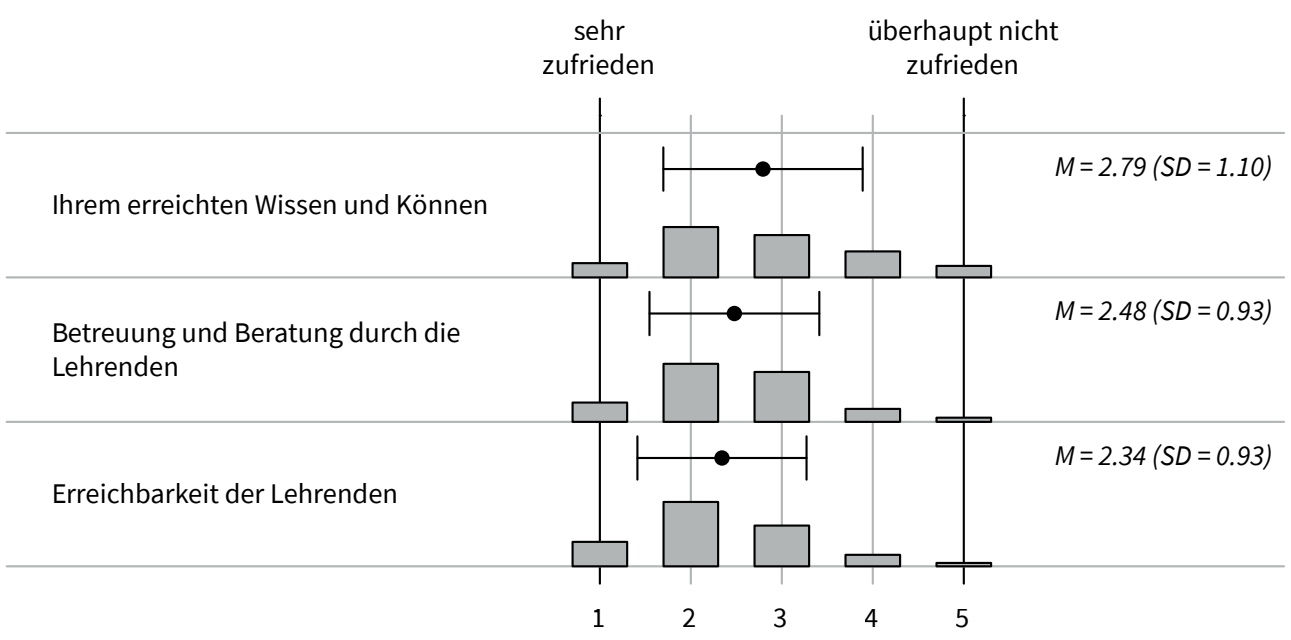

Abb. 10.: Zufriedenheit mit ausgewählten Aspekten. 
Die Herausforderungen in der aktuellen Studiensituation führten in der Gesamtschau aber offenbar nicht dazu, dass sich Studierende mit dem Gedanken eines Studienabbruchs tragen - nur knapp $7 \%(n=144)$ geben an, dies in Erwägung zu ziehen. Auf die Frage nach den Gründen für die Abbruchsintention führen dann allerdings knapp die Hälfte der Befragten coronaspezifische Gründe an, gefolgt von persönlichen und studienbezogenen Gründen (s. Tab. 7).

\begin{tabular}{|c|c|c|c|c|c|}
\hline $\begin{array}{l}\text { Aus welchem Grund oder } \\
\text { Gründen, werden Sie das Stu- } \\
\text { dium (vielleicht) abbrechen? }\end{array}$ & Grund & Bachelor & Master & $\begin{array}{l}\text { Phi-Koeff: } \\
\text { Korr. zw. } \\
\text { Unter- } \\
\text { stüztzung } \\
\text { und Ab- } \\
\text { schlussart }\end{array}$ & p-Wert \\
\hline $\begin{array}{l}\text { Coronaspezifische Gründe (z. B. } \\
\text { digitales Semester) }\end{array}$ & $46.5 \%$ & $43.5 \%$ & $54.5 \%$ & & n.s. \\
\hline $\begin{array}{l}\text { Persönliche Gründe (z. B. priva- } \\
\text { te Probleme, keine Motivation) }\end{array}$ & $41.7 \%$ & $38.0 \%$ & $51.5 \%$ & & n.s. \\
\hline Leistungsdruck zu hoch & $34.0 \%$ & $37.0 \%$ & $27.3 \%$ & & n.s. \\
\hline Prüfung/en nicht bestanden & $34.0 \%$ & $38.0 \%$ & $24.2 \%$ & & n.s. \\
\hline $\begin{array}{l}\text { Meine Erwartungen an das } \\
\text { Studium wurden nicht erfüllt }\end{array}$ & $34.0 \%$ & $30.6 \%$ & $42.4 \%$ & & n.s. \\
\hline Anforderungen zu hoch & $27.1 \%$ & $29.6 \%$ & $21.2 \%$ & & n.s. \\
\hline $\begin{array}{l}\text { Studium ist zeitlich nicht } \\
\text { erfüllbar }\end{array}$ & $22.9 \%$ & $21.3 \%$ & $24.2 \%$ & & n.s. \\
\hline Finanzielle Gründe & $18.8 \%$ & $15.7 \%$ & $24.2 \%$ & & n.s. \\
\hline Fehlende Berufsperspektive & $13.2 \%$ & $11.1 \%$ & $18.2 \%$ & & n.s. \\
\hline Gesundheitliche Gründe & $11.1 \%$ & $11.1 \%$ & $9.1 \%$ & & n.s. \\
\hline $\begin{array}{l}\text { Familiäre Gründe (z. B. } \\
\text { Pflege oder Betreuung von } \\
\text { Angehörigen) }\end{array}$ & $7.6 \%$ & $4.6 \%$ & $18.2 \%$ & 0.214 & 0.011 \\
\hline $\begin{array}{l}\text { Zusage einer anderen } \\
\text { Universität }\end{array}$ & $2.8 \%$ & $0.9 \%$ & $9.1 \%$ & 0.208 & 0.013 \\
\hline
\end{tabular}

Tab. 7.: Gründe für Abbruchintentionen (Mehrfachantworten möglich).

Vergleicht man Studierende, die sich Gedanken über einen Studienabbruch machen, mit Studierenden, die laut Selbstauskunft keine derartigen Gedanken hegen, hinsichtlich ihrer persönlichen Eingangsvoraussetzungen, so ergibt sich folgendes Bild: Studierende ohne Studienabbruchgedanken haben ein höheres beständiges Interesse (Hedges $g=0.50$ ), eine höhere Beharrlichkeit (Hedges $g=0.42$ ), eine höhere Selbstkontrolle und Zielverfolgung (Hedges $g=0.71$ ), eine höhere Selbstmotivierung und Emotionskontrolle (Hedges $g=0.54$ ), eine geringere Tendenz zur Prokrastination (Hedges $g=0.54$ ), höhere gefühlsbezogene Valenzen des Studiums (Hedges $g=0.44$ ) 
sowie höhere persönliche wertbezogene Valenzen des Studiums (Hedges g $=0.47$ ). Zudem sind sie zufriedener mit ihrem erreichten Wissen und Können (Hedges $\mathrm{g}=0.76)$ im Vergleich zu den Studierenden mit Studienabbruchsgedanken.

\section{Konsequenzen für eine agile Qualitätsentwicklung der Hochschullehre}

Die Ergebnisse der Studie geben Einblicke in die studentische Wahrnehmung der Qualität und Effekte des durch die Pandemie forcierten reinen Digitalsemesters an der Universität Stuttgart und erlauben zudem erste Rückschlüsse bezüglich spezifischer Unterstützungsbedarfe, die freilich angesichts des Standortbezugs der Datengrundlage und der fehlenden Repräsentativität der selbstselektiven Stichprobe zurückhaltend zu bewerten sind. Bezüglich der technischen Eingangsvoraussetzungen zeichnen sich zu Beginn des Digitalsemesters auf Studierendenseite keine grösseren Defizite oder Engpässe ab - die Befragten sind hinreichend ausgestattet und verfügen über die notwendige Vertrautheit im Umgang mit den für das digitale Studium notwendigen Computeranwendungen. Nur hinsichtlich der psychoregulatorischen und emotionalen Eingangsvoraussetzungen (Selbststeuerungsfähigkeit, IT-bezogene Selbstwirksamkeit, Beharrlichkeit und Interesse) zeigen sich kleinere Unterschiede zugunsten der Masterstudierenden. Die Erwartungen bezüglich der Gestaltung der digitalen Lehrformate sind im Wesentlichen auf die Bereitstellung von Audio- und Videodateien in guter technischer Qualität als Ersatz für die entfallende Präsenzlehre und den Austausch mit den Lehrenden gerichtet. Nach Abschluss der Vorlesungszeit fällt die erste Bilanz der Studierenden gemischt aus - während die soziale Isolation und ihre Auswirkungen auf das Studierverhalten und Wohlbefinden sich insbesondere für Bachelorstudierende eher als problematisch herausstellen, werden die zeitliche Flexibilität und die insgesamt zufriedenstellende Gestaltungsqualität der digitalen Lehre durchaus geschätzt. Optimierungsbedarf wird insbesondere hinsichtlich der mangelnden Verfügbarkeit von Literatur aufgrund der Bibliotheksschliessung und der Veranstaltungs- und Prüfungsorganisation gesehen. Nach Abschluss der Prüfungsphase im Herbst 2020 zeigt sich, dass knapp die Hälfte der Studierenden weniger Prüfungen erbracht hat als ursprünglich geplant und die Gründe dafür durchaus auch in der spezifischen pandemiebedingten Studiensituation zu suchen sind. Nichtsdestoweniger führte die erschwerte Situation bei dem Gros der Befragten nicht unmittelbar zu der Intention des Studienabbruchs, sondern allenfalls zu zeitlichen Verzögerungen des Studienablaufs. Insgesamt schliessen die Ergebnisse insofern gut an die bisherige Befundlage an, deuten jedoch daraufhin, dass bezüglich der sichtbaren psycho-emotionalen Belastungen, denen die Studierenden ausgesetzt waren, im Folgenden weiterführende Analysen angezeigt sind, um noch gezielter gruppenspezifische Risikofaktoren adressieren zu können. Zudem stellen 
sich Fragen nach konkreten hochschuldidaktischen Implikationen und strategischen Konsequenzen der Ergebnisse für die Weiterentwicklung der hochschulischen Lehrqualität.

Im Kontext der Ad-hoc-Transformation zur digitalen Lehre folgte die Universität Stuttgart dem Prinzip des agilen Managements (Baecker 2017): Bedarfe der Studierenden antizipieren, pragmatisch und flexibel auf Sicht fahren (Lehner und Volk 2018) und dabei regelmässig überprüfen, inwieweit das Angebot der digitalen Lehre Erfolg hat oder steuernder Eingriffe bedarf. Die Studierenden als Gruppe sind nicht nur in fachlicher Hinsicht heterogen, sondern auch mit Blick auf ihre Lernvoraussetzungen, ihre häusliche Umgebung und ihre Studienerfahrung an der Universität Stuttgart. Aus unserer Studie sind zunächst mehrere konkrete hochschuldidaktische Implikationen abzuleiten, die sich auf das Prinzip der Evaluation einer Lehrangebots aus der Sicht der Studierenden als dessen Rezipienten stützt und weiterführende Erwägungen bezüglich zielführender Digitalisierungsstrategien erlaubt.

Für Bachelorstudierende mit relativ wenig Studienerfahrung am aktuellen Studienort Stuttgart ist die Festigung einer studentischen Sozialisation besonders in den ersten Semestern zentral. Sozialisation im Hochschulkontext folgt grundsätzlich einem Ansatz, indem Studierende sich vernetzen und wesentliche Informationen nicht "von oben», sondern von ihren Peers erhalten, etwa aus Fachschaften, Lerngruppen oder Tutorien. Baecker (2017) bezeichnet dieses Prinzip als den Wechsel «von Silos auf Netzwerke» (ebd., 19). Die Universität kann hier Rahmenbedingungen schaffen, etwa eine Matchingbörse für die Suche nach Lerngruppen oder Mentorinnen und Mentoren für Neuankömmlinge, die sicherstellen, dass alle relevanten Informationen vermittelt werden. Ein weiteres Beispiel ist die Vorbereitung auf Prüfungen, die begleitend zu den Lehrveranstaltungen im Peerkontext stattfindet.

Nimmt man den Agilitätsansatz ernst und begreift Studierende als rückmeldende Kundschaft, so sollte auch die selektive Beibehaltung des Digitalen für die Semester nach dem Ende der pandemiebedingten Vorgaben im Diskurs mit den Studierenden angegangen werden. In Zeiten der Quantifizierung von Lernzeit und Aufwand in 30-Stunden-Paketen zur Umrechnung in ECTS sind auch Potenziale einer veränderten Nutzung von Präsenzzeit, etwa die Vermittlung von überfachlichen Kompetenzen wie Selbststeuerungsfähigkeit, an der Universität zu berücksichtigen, die jedoch nur dann in effiziente Lernzeit mündet, wenn die Bedarfe und Voraussetzungen der Studierenden in die Gestaltung einbezogen werden. Agil heisst an dieser Stelle insofern, Studierende als kontinuierliche und wertvolle Quelle von Feedback in die formative Evaluation der digitalen Lehre einzubinden und jenen Rückmeldungen und Evidenzen systematisch zu begegnen.

Die Rückmeldung der Studierenden zeigte klare Präferenzen für Formate oder Medien und deren Eignung für digitale Lehre an der Universität. Diese Präferenzen müssen den Lehrenden kommuniziert werden und bei Bedarf ist hochschuldidaktischer 
Support nötig, etwa für die Nutzung noch unbekannter Tools oder für die Konzeption neuer didaktischer Settings für bekannte und bisher analog gelehrte Inhalte. Hier ist eine erneute Rückkopplung an die Studierenden sinnvoll, um bedarfsgerecht in die Semesterplanung zu gehen.

Abgesehen von diesen konkreten Implikationen ziehen wir aus unserer Studie und den Befunden aus der Perspektive der Studierenden den strategischen Schluss, dass Qualitätsentwicklung im Bereich der Lehre nicht zuletzt aufgrund der in der Regel geringen Rückläufe kaum eine andere Wahl hat, als agil an das Thema heranzugehen: pragmatisch und kurzfristig Daten sammeln, auswerten und gemeinsam mit den relevanten Akteursgruppen interpretieren - dies ist noch im laufenden Semester im Rahmen der Taskforce Digitale Lehre und in konstruktivem Dialog erfolgt. Qualitätsentwicklung ist insofern per se ein dynamischer Prozess, der wesentlich eher in Form von Netzwerken innerhalb der Hochschule auszugestalten ist als top-down (Baecker 2017). Die Studierenden als Gruppe mit spezifischen Eingangsvoraussetzungen und Bedürfnissen verändern sich konstant, so dass eine der Lehren aus dem ersten Corona-Semester 2020 für die Universität Stuttgart ist, dass Agilität als Managementansatz sehr tragfähig für innovative und dynamische Organisationen wie Hochschulen ist, wenn sich auch gegenüber Organisationen mit Gewinnerzielungsinteresse freilich systematische Unterschiede ergeben. Agilität und Evidenzbasierung sind ungeachtet dessen zentrale strategische Momente in der Überprüfung und Weiterentwicklung der Qualität der Hochschullehre, die sich nicht zuletzt in disruptiven Transformationsprozessen bewähren. Hervorzuheben bleibt aber auch, dass dazu eine möglichst partizipative und mehrperspektivische Bestimmung der Zielkriterien und Indikatoren erfolgreicher digitaler Hochschullehre erfolgen sollte (Wannemacher 2017).

In der Gesamtschau bleibt zu konstatieren, dass Hochschulen sich im besten Fall als permanente Forschungs- und Entwicklungsprojekte (Mormann und Willjes 2013) begreifen, die dennoch in Ergänzung ihrer evidenzbasierten und agilen Perspektive auf die Digitalisierung der Hochschullehre auch normative Fragen nach dem Stellenwert des Bildungsgedankens und damit einem möglichen Spannungsverhältnis zwischen Qualität und Effizienz in ihren Digitalisierungsstrategien kritisch durchdenken sollten (Bedenlier und Deimann 2020). Dies wird besonders mit Blick auf die sich aktuell vollziehende Entwicklung relevant, die den verstärkten Einsatz von Data Analytics und künstlicher Intelligenz zur prozessnahen Diagnose und Förderung individueller Lehr-Lern-Prozesse mit sich bringt (Seufert, Guggemos, und Sonderegger 2020) und zu neuen Mensch-Maschine-Interaktionen und grundlegenden Veränderungen der Hochschuldidaktik führen wird, die umfassend reflektiert sein wollen. 


\section{Literatur}

Adedoyin, Olasile Babatunde, und Emrah Soykan. 2020. "Covid-19 pandemic and online learning: the challenges and opportunities». Interactive Learning Environments. https://doi.or g/10.1080/10494820.2020.1813180.

Baecker, Dirk. 2017. «Agilität in der Hochschule». Die Hochschule 1: 19-28. https://hsdbs.hof. uni-halle.de/documents/t2388.pdf.

Barends, Eric, Denise M. Rousseau, und Rob B. Briner. 2014. Evidence-Based Management: The Basic Principles. Amsterdam: Centre for Evidence-Based Management. https://research. vu.nl/ws/portalfiles/portal/42141986/complete+dissertation.pdf.

Bedenlier, Svenja, und Markus Deimann. 2020. «Bildung und Digitalisierung im Spiegel von Digitalisierungsstrategien». Zeitschrift für Hochschulentwicklung 15(1): 41-59. https://doi. org/10.3217/zfhe-15-01/03.

Benton Stephen L., und William E. Cashin. 2014. «Student Ratings of Instruction in College and University Courses». In Higher Education: Handbook of Theory and Research, herausgegeben von Michael Paulsen, und Laura Perna. Springer: Dordrecht. https://doi.org/10.1007/97894-017-8005-6_7.

Bond, Melissa, Victoria I. Marin, Carina Dolch, Svenja Bedenlier, und Olaf Zawacki-Richter. 2018. "Digital transformation in German higher education: student and teacher perceptions and usage of digital media». International Journal of Educational Technology in Higher Education 15 (48). https://doi.org/10.1186/s41239-018-0130-1.

Bortz, Jürgen, und Christof Schuster. 2010. Statistik für Human- und Sozialwissenschaftler (7. vollständig überarbeitete und erweiterte Auflage). Berlin: Springer. https://link.springer. com/book/10.1007/978-3-642-12770-0.

Brahm, Taiga, Tobias Jenert, und Dieter Euler. 2016. Pädagogische Hochschulentwicklung: Von der Programmatik zur Implementierung. Berlin: Springer. https://doi.org/10.1007/978-3658-12067-2.

Brahm, Taiga, und Marina Pumptow. 2021. «Students' Digital Media Usage at the University of Tübingen During the CoViD19 Semester 2020 Compared to 2018». MedienPädagogik 40 (CoViD-19): 118-137. https://doi.org/10.21240/mpaed/40/2021.11.13.X.

Dittler, Ullrich. 2017. «Ein kurzer historischer Rückblick auf die bisherigen drei Wellen des ELearning». In E-Learning 4.0. Mobile Learning, Lernen mit Smart Devices und Lernen in Sozialen Netzwerken, herausgegeben von Ullrich Dittler. Berlin: De Gruyter, 5-42. https://doi. org/10.1515/9783110468946.

Dittler, Ullrich, und Christian Kreidl. 2020. Ergebnisse einer trinationalen Umfrage unter Studierenden aus Deutschland, Österreich und der Schweiz zu ihren Erfahrungen mit der Online-Lehre des Sommersemesters 2020. https://nbn-resolving.org/urn:nbn:de:bsz:fn1opus4-67375.

Duckworth, Angela, Christopher Peterson, Michael Matthews, und Dennis Kelly. 2007. "Grit: Perseverance and passion for long-term goals». Journal of Personality and Social Psychology 92(6): 1087-1101. https://doi.org/10.1037/0022-3514.92.6.1087. 
Egloffstein, Marc, und Dirk Ifenthaler. 2021. «Tracing Digital Transformation in Educational Organizations from Individual to Organizational Perspectives». In Digital Transformation of Learning Organizations, herausgegeben von Dirk Ifenthaler, Sandra Hofhues, Marc Egloffstein und Christian Helbig. Cham: Springer. 41-57. https://doi.org/1007/978-3-030-558789_3.

Euler, Dieter, und Sabine Seufert. 2005. «Von der Pionierphase zur nachhaltigen Implementierung - Facetten und Zusammenhänge einer pädagogischen Innovation». In E-Learning in Hochschulen und Bildungszentren, herausgegeben von Dieter Euler und Sabine Seufert, 1-24. E-Learning in Wissenschaft und Praxis 1. München: De Gruyter Oldenbourg. https:// doi.org/10.1515/9783486593754.1.

Getto, Barbara, und Michael Kerres. 2017. «Akteurinnen/Akteure der Digitalisierung im Hochschulsystem: Modernisierung oder Profilierung?». Zeitschrift für Hochschulentwicklung 12(1): 123-142. https://doi.org/10.3217/zfhe-12-01/07.

Hafer, Jörg, Peter Kostädt, und Ulrike Lucke. 2021. «Das Corona-Virus als Treiber der Digitalisierung? Eine kritische Analyse am Beispiel der Universität Potsdam». In Wie Corona die Hochschullehre verändert, herausgegeben von Ullrich Dittler und Christian Kreidl, Springer Nature, 219-242. https://doi.org/10.1007/978-3-658-32609-8_15.

Handke, Jürgen. 2020. Handbuch Hochschullehre Digital: Leitfaden für eine moderne und mediengerechte Lehre. 3. Aufl. Marburg: Tectum Wissenschaftsverlag.

Hodges, Charles, Stephanie Moore, Barb Lockee, Torrey Trust, und Aaron Bond. 2020. «The Difference Between Emergency Remote Teaching and Online Learning». EDUCAUSE. https:// er.educause.edu/articles/2020/3/the-difference-between-emergency-remote-teachingand-online-learning.

Hofhues, Sandra, Mandy Schiefner-Rohs, Sandra Aßmann, und Taiga Brahm. 2020. Studierende - Medien - Universität. Einblicke in studentische Medienwelten. Münster: Waxmann. https:// doi.org/10.31244/9783830990499.

Krapp, Andreas, Ulrich Schiefele, Klaus Peter Wild, und Adolf Winteler. 1993. «Der Fragebogen zum Studieninteresse (FSI)». Diagnostika 39 (1993) 4: 335-351. https://psycnet.apa.org/record/1994-86134-001.

Kopp, Michael, Ortrun Gröblinger, und Simone Adams. 2019. Five common assumptions that prevent digital transformation at higher education institutions. NTED2019 Proceedings, 1448-1457. https://library.iated.org/view/KOPP2019FIV.

Lehner, Marion, und Benno Volk. 2018. «Agiles Evaluationskonzept zur evidenzorientierten Qualitätsentwicklung in der Hochschullehre». Zeitschrift für Hochschulentwicklung 13(1): 253-273. https://doi.org/10.3217/zfhe-13-01/13.

Leimeister, Jan Marco, und Klaus David. 2019. Chancen und Herausforderungen des digitalen Lernens. Methoden und Werkzeuge für innovative Lehr-Lern-Konzepte. Springer. https://doi. org/10.1007/978-3-662-59390-5. 
Mormann, Hannah, und Kristina Willjes. 2013. «Organisationsprojekt und Projektorganisation. Softwareeinführungsprojekte in Hochschulen aus einer organisationssoziologischen Perspektive». In IT und Organisation in Hochschulen, herausgegeben von Friedrich Statmann. HIS: Forum Hochschule. https://his-he.de/fileadmin/user_upload/Publikationen/Forum_ Hochschulentwicklung/fh-201304.pdf.

Mulders, Miriam, und Sophia Krah. 2021. «Digitales Lernen während der Covid-19-Pandemie aus Sicht von Studierenden der Erziehungswissenschaften. Handlungsempfehlungen für die Digitalisierung von Hochschullehre». Medienpädagogik 40 (CoViD-19): 25-44. https:// doi.org /10.21240/mpaed/40/2021.01.29.X.

Rapanta, Chrysi, Luca Botturi, Peter Goodyear, Lourdes Guàrdia, und Marguerite Koole. 2020. "Online University Teaching During and After the Covid-19 Crisis: Refocusing Teacher Presence and Learning Activity». Postdigital Science and Education. https://doi.org/10.1007/ s42438-020-00155-y.

Richter, Tobias, Johannes Naumann, und Norbert Groeben. 2001. «Inventar zur Computerbildung (INCOBI). Ein Instrument zur Erfassung von Computer Literacy und computerbezogenen Einstellungen bei Studierenden der Geistes- und Sozialwissenschaften». Psychologie in Erziehung und Unterricht 48: 1-13.

Rheinberg, Falko, und Mirko Wendland. 2003. Itemübersicht zum Fragebogen SSI-K-32 (Selbststeuerungs-Inventar-Kurzform). Universität Potsdam, Institut für Psychologie.

Rodabaugh, Rita Cobb, und David A. Kravitz. 1994. «Effects of Procedural Fairness on Student Judgments of Professors». Journal on Excellence in College Teaching 5 (2): 67-83.

Schmid, Ulrich, Lutz Goertz, Sabine Radomski, Sabrina Thom, und Julia Behrens. 2017. Monitor Digitale Bildung. Die Hochschulen im digitalen Zeitalter. https://www.bertelsmann-stiftung.de/fileadmin/files/BSt/Publikationen/GrauePublikationen/DigiMonitor_Hochschulen_final.pdf.

Schmidt, Fabian, Johanna Fleckenstein, Jan Retelsdorf, Lauren Eskreis-Winkler, und Jens Möller. 2019. «Measuring grit: A German validation and a domain-specific approach to grit». European Journal of Psychological Assessment 35 (3): 436-447. https://doi.org/10.1027/1015$5759 / a 000407$.

Schyns, Birgit, und Gernot von Collani. 2002. «A new occupational self-efficacy scale and its relation to personality constructs and organizational variables». European Journal of Work and Organizational Psychology 11 (2): 219-241. https://doi.org/10.1080/13594320244000148.

Seufert, Sabine, Martin Ebner, Michael Kopp, und Bettina Schlass. 2015. «Editorial: E-LearningStrategien für die Hochschullehre». Zeitschrift für Hochschulentwicklung 10 (2): 9-18. https://doi.org/10.3217/zfhe-10-02/01.

Seufert, Sabine, Josef Guggemos, und Luca Moser. 2019. «Digitale Transformation in Hochschulen: auf dem Weg zu offenen Ökosystemen». Zeitschrift für Hochschulentwicklung 10 (2): 85-107. https://doi.org/10.3217/zfhe-14-02/05. 
Seufert, Sabine, Josef Guggemos, und Stefan Sonderegger. 2020. «Digitale Transformation der Hochschullehre: Augmentationsstrategien für den Einsatz von Data Analytics und Künstlicher Intelligenz». Zeitschrift für Hochschulentwicklung 15 (1): 81-101. https://doi. org/10.3217/zfhe-15-01/05.

Skulmowski, Alexander, und Günter Daniel Rey. 2020. «COVID-19 as an accelerator for digitalization at a German university: Establishing hybrid campuses in times of crisis». Human Behavior and emerging Technologies 2 (3): 212-216. https://doi.org/10.1002/hbe2.201.

Suwalski, Petra. 2020. Systemakkreditierung an Hochschulen. Anforderungen, Maßnahmen und Effekte aus der Perspektive von Hochschulakteuren. Opladen: Budrich Academic Press. https://doi.org/10.2307/j.ctv153k6tn.

Wannemacher, Klaus. 2017. «Digitalisiertes Lehren und Lernen als organisationales Problem in den deutschen Hochschulen». Die Hochschule 1, 99-110. https://doi.org/10.25656/01:16639.

Zierer, Klaus. 2018. Lernen 4.0: Pädagogik vor Technik - Möglichkeiten und Grenzen einer Digitalisierung im Bildungsbereich. Schneider Verlag Hohengehren. 